Portland State University

PDXScholar

7-9-2021

\title{
Simulation of Light Propagation Captured by Photoemission Electron Microscopy (PEEM)
}

Nabila Islam

Portland State University

Follow this and additional works at: https://pdxscholar.library.pdx.edu/open_access_etds

Part of the Nanoscience and Nanotechnology Commons, and the Optics Commons Let us know how access to this document benefits you.

\section{Recommended Citation}

Islam, Nabila, "Simulation of Light Propagation Captured by Photoemission Electron Microscopy (PEEM)" (2021). Dissertations and Theses. Paper 5722.

https://doi.org/10.15760/etd.7595

This Thesis is brought to you for free and open access. It has been accepted for inclusion in Dissertations and Theses by an authorized administrator of PDXScholar. Please contact us if we can make this document more accessible: pdxscholar@pdx.edu. 
Simulation of Light Propagation Captured by

Photoemission Electron Microscopy (PEEM)

by

Nabila Islam

A thesis submitted in partial fulfillment of the requirements for the degree of

Master of Science

in

Physics

Thesis Committee:

Rolf Könenkamp, Chair

Erik Sánchez

Jay Louise Nadeau

Portland State University

2021 


\begin{abstract}
The Photoemission electron microscopes (PEEM) is a powerful tool capable of synchronously imaging wave nature of light manifested by interference patterns as well as its particle nature through the energy exchange between the incident photons and the photoemitted imaging electrons. PEEM offers a non-invasive high-resolution approach for studying light propagation and interaction phenomena within a nanophotonic waveguide $[7,8]$. The electric field intensity variation of the interference pattern yielded by the interaction between the incident light and the guided mode coupled into the waveguide produces varying photoemission yields creating contrast in PEEM image. The guided modes cannot be excited simply by exposing the waveguide surface to light. A compact efficient coupler such as diffraction gratings is required. In this work an efficient grating coupler is designed using Finite Element Analysis (FEA) to couple a pump beam of $400 \mathrm{~nm}$ wavelength from a Ti:sapphire laser source into a $180 \mathrm{~nm}$ thick ITO waveguide on a $200 \mathrm{~nm}$ thick glass substrate. The PEEM image of the electric field distribution yielded by the interference between the guided mode and a probe pulse was simulated and the properties of the interference pattern was studied. The phase delay between the pump and the probe pulse was then increased in steps of $\pi / 2$ for one optical cycle of the excitation laser causing the interference pattern on the ITO surface to progress in position. By simulating the image of the interference pattern for each delay step and by compiling the images a movie-like documentation of the light propagation in the waveguide is obtained. Simulations show the interference maxima peaks to move away from the coupler with the speed of light for an ITO waveguide.
\end{abstract}




\section{Acknowledgements}

I would like to express my sincere gratitude to my research advisor and the chair of my master's thesis committee Dr. Rolf Könenkamp for providing me the opportunity to conduct research in Portland State University and for guiding me throughout this research. I would also like to thank Dr. Erik Sànchez and Dr. Jay Nadeau for serving as the members of my master's thesis committee. Additionally, I would like to thank Dr Theodore Stenmark, Christopher Scheffler and Dr. Robert Word for helping me to learn and troubleshoot software for simulation and data analysis and for providing me with necessary resources.

I am extremely grateful to my mother Nasima Islam and father Dr. M. Aminul Islam for their love, care and sacrifices for providing me all the opportunities for education and preparing me for the future. I am also very thankful to my husband Dr. Saad Ahmed for providing unending inspiration, support and encouragements for this work. 


\section{Table of Contents}

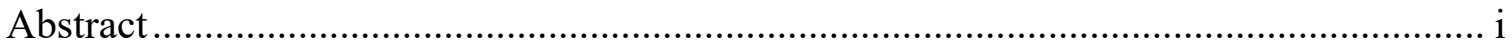

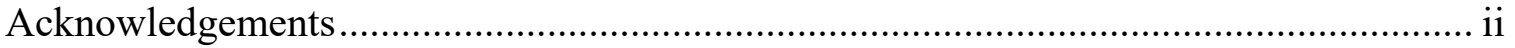

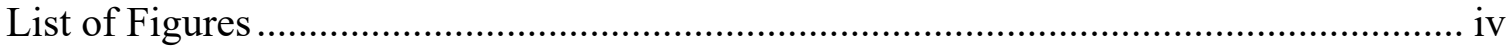

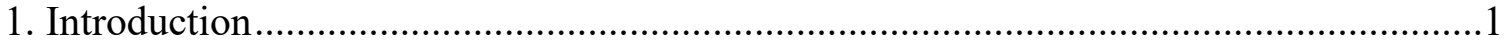

2. Photoemission Electron Microscopy (PEEM) ..............................................................4

2.1 Theory and working principle of PEEM: ....................................................

2.2 Sample setup and experiment using PEEM: ...............................................

3. Coupled mode analysis for waveguides............................................................... 11

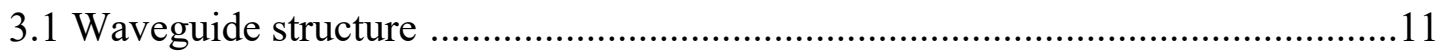

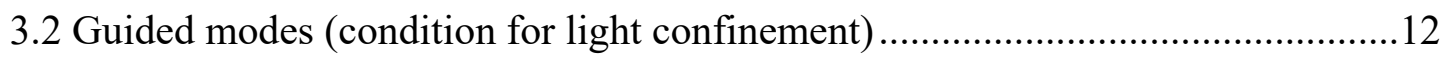

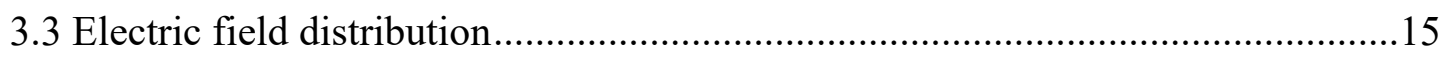

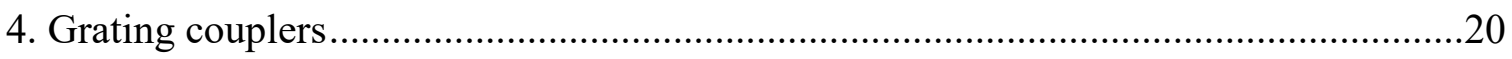

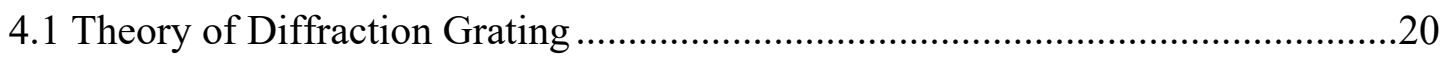

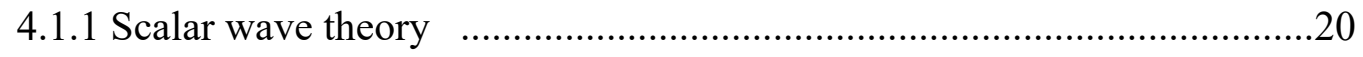

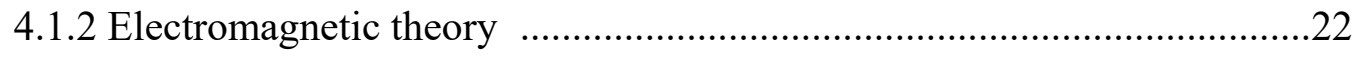

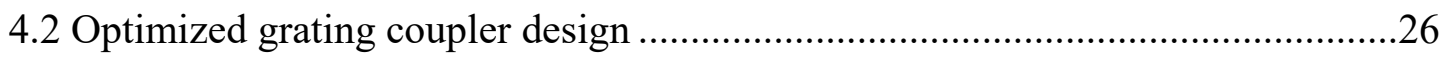

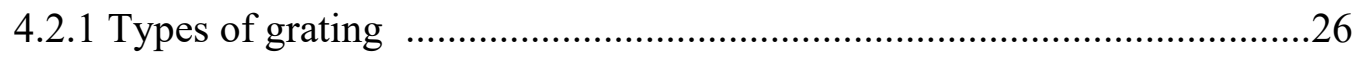

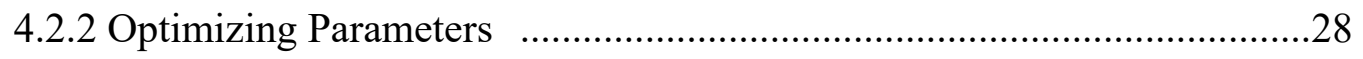

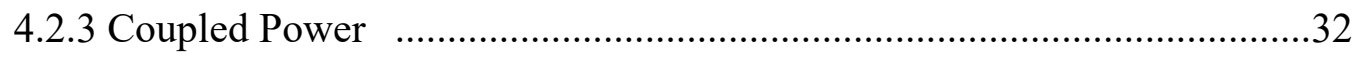

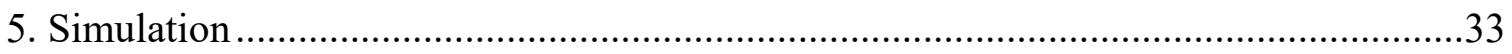

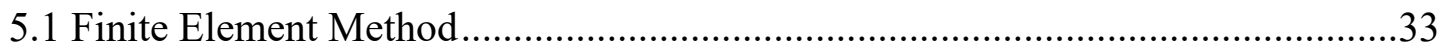

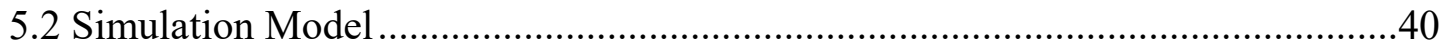

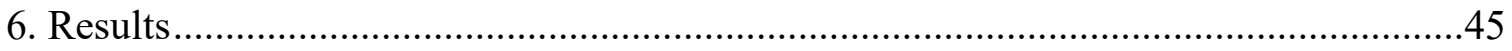

6.1 Fourier Transform Verifying Mode Coupling …..............................................50

6.2 Grating Length, Depth and Coupled Power ......................................................53

6.3 Grating Shape and Coupled Power ....................................................................54

6.4 Phase Delay and Interference Pattern Position ..................................................55

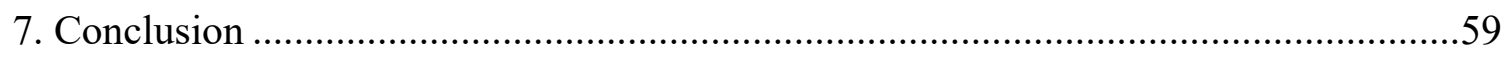

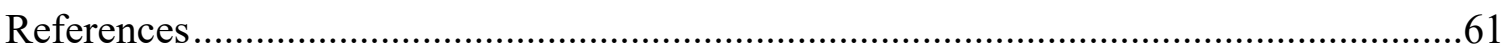




\section{List of figures}

Figure 2.1: Schematic diagram of PEEM. [15] .8

Figure 2.2: Schematic diagram of PEEM sample chamber containing the waveguide illuminated by the pump and the probe beam.[15] .................................. 10

Figure 3.1: Schematic representation of planar waveguide. .........................12

Figure 3.2: Wave propagation in the waveguide. ................................13

Figure 3.3: Discrete modes with different $m$ values within the waveguide .............15

Figure 3.4: The graphical solution for the propagation constant of an ITO waveguide of $290 \mathrm{~nm}$ thickness and 2.14 refractive index with air superstrate and glass substrate with refractive index 1.5 at a wavelength of $410 \mathrm{~nm}$. The propagation constants are determined from the non-asymptotic intersections. 20

Figure 4.1: transmitted and reflected orders of a binary diffraction grating. .............23

Figure 4.2: (a) binary grating, (b) blazed grating and (c) sinusoidal grating. ...........28

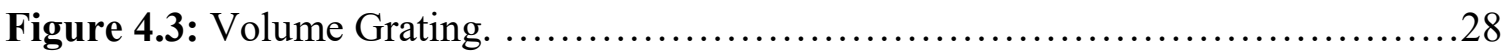

Figure 4.4: waveguide coupling with normally incident beam........................30

Figure 4.5: Free space coupling. .................................................

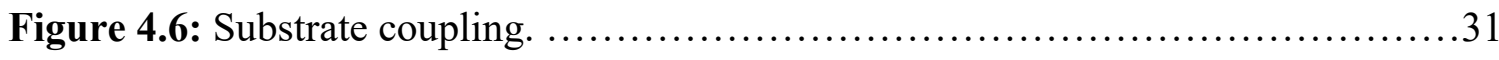

Figure 4.7: Waveguide coupling for oblique incident angle. .........................33

Figure 5.1: edge and node triangular elements. ..................................... 37

Figure 5.2: COMSOL desktop environment. .....................................41

Figure 5.3: Triangular meshing throughout the model. .............................43

Figure 5.4: Electric field distribution image result. ................................44

Figure 5.5: Power flow calculation through the blue and red lines across ITO thickness.

Figure 6.1: (a) The oblique beam incident at $60^{\circ}$ angle on the grating etched on the ITO surface in the air medium that excites a single mode that interferes with the normal beam incident on the bottom surface of ITO in glass medium (b) Close up of the ITO layer showing interference pattern created by the guided mode and the normal beam on the left side of the grating. (C) Line graph of the electric field distribution of the interference pattern on the ITO surface extracted from the left side of the grating. 
Figure 6.2 In the COMSOL model of the sample two rectangular shapes has been cut out (with no medium assigned to it) in the air medium to simulate obstacle preventing interference between the obliquely incident beam and the guided mode. .50

Figure 6.3: The wave front of the obliquely incident beam upon incident on the grating excites the guided mode that interferes with the wave front of the normally incident beam. .52

Figure 6.4: Fourier transform of normalized electric field distribution extracted for (a) both normal and oblique beam incident on the sample where the oblique beam only excites the guided mode and only the normal beam interfering with the mode and (b) only the normal beam incident on the sample exciting the mode as well as interfering with it.

Figure 6.5: The oblique beam incident on the optimized grating coupler excites a guided mode that propagates away from the grating within the waveguide.

Figure 6.6: graph showing the variation of coupled power with grating size at different grating depths. .55

Figure 6.7: graph showing comparison of coupled power for different grating shapes. The depths are chosen for each shape to achieve maximum coupled power. .56

Figure 6.8: Simulated image of electric field distribution on the ITO surface as well as within the ITO layer. Each dashed line shows the edge of the same peak progressing with increased time

delay.

Figure 6. 9: Line electric field distribution for each delay steps across the ITO surface showing time progression of the electric field distribution peaks. Each dashed line indicates the same peak advancing in distance in each successive frame. 


\section{Introduction}

Optical integrated circuits utilize photons for the transmission and processing of signals. The advantages of using photons include larger bandwidth, minimal loss and electromagnetic interference and lower required powers. As a result, integrated photonics is finding use in wide range of areas including fiber optic communication [4], quantum communication and computation [1], defense [2], sensors [5], biomedical devices [6] and many others. The advancement of integrated photonics is achieved through the development of the optical components such as light modulators, detectors, multiplexers, waveguides and couplers.

In optical integrated circuits, light propagates as guided modes within a dielectric waveguide. The light remains confined within the waveguide by means of total internal reflection from the waveguide boundary with the lower refractive index substrate (glass) and superstrate (air). These modes can interfere with a probe beam incident on the waveguide surface and create stationary interference patterns in and outside the sample. The field distribution yielded by this interference carries direct information about the wave propagation in the waveguide and, in addition, information about the specimen's optical properties.

If the time delay between the mode guided within the waveguide and the probe beam is changed, the position of the interference maxima and minima will also be changed as a consequence of the propagation of the guided mode. Thus, by gradually increasing the time delay through a complete optical cycle we can observe the motion of 
the superposition pattern along the direction of the mode's propagation. Assuming a refractive index of 2 , light at a wavelength of $410 \mathrm{~nm}$ propagates a distance of $205 \mathrm{~nm}$ in one optical cycle. To be able to track this propagation in a single cycle in 4 steps, we need a spatial resolution of $\sim 50 \mathrm{~nm}$ and delay times of 170 as. These parameters can indeed be realized in PEEM.

The electromagnetic field distribution created by the mode and probe interaction is equivalent to a photon distribution which causes electron emission by means of the photoelectric effect. Some of the photoelectrons are emitted into the vacuum above the waveguide are collected in the PEEM to form an image. This electron image will represent the photonic interference pattern in the waveguide.

In this thesis we want to analyze through simulation how the time propagation of the guided modes can be imaged using PEEM.

We simulate a nanophotonic waveguide consisting of a thin ITO layer embedded between a glass substrate and vacuum and situated within the sample chamber of PEEM where it is illuminated. The incident light is passed through a beam splitter that splits it into two separate beams one of which is incident on the top ITO surface obliquely and the other one is incident on a mirror placed below the waveguide. This second beam gets reflected by the mirror and is incident on the bottom surface of the waveguide perpendicularly to that surface. The oblique beam excites a guided mode that propagates through the waveguide. The normal beam goes through a delay stage before entering the waveguide from below and interferes with the propagating mode. The delay between the oblique beam and the normal beam is varied in gradual steps and the PEEM image of the 
interference patterns created for each of the delay steps serve as snapshot of the time evolution of the waveguide mode. These snapshots are compiled into a movie. Analysis of such time progression of the waveguide mode and its field distribution provides us insight into the dynamic properties of an integrated photonic circuit.

For coupling incident light beam into the waveguide and creating the guided modes, a grating coupler is also designed through numerical simulation. The PEEM setup allows the pump beam to incident on the sample obliquely at an angle greater than or equal to $60^{\circ}$. The grating is used to couple the incident beam more efficiently in the reverse direction. In the process of coupling the light into the waveguide, a diffraction pattern is generated. This pattern is caused by an interference between the oblique excitation beam and its diffracted wave, but there is no delay stage between the two partial beams. While the mode pattern in the waveguide propagates with time, the diffraction pattern remains stationary. The addition of this stationary pattern makes it difficult to quantify the moving pattern. However, the diffraction pattern is usually limited in size, and it is therefore possible to observe the movie outside the diffraction pattern when the location is appropriately chosen. In the reverse direction the diffraction pattern has a shorter period than in the forward direction. For this reason, coupling in the reverse direction is desirable [9] for our purpose. While the coupling of a particular mode is done by adjusting the grating period in accordance with the mode's effective index, the coupled power of the mode varies with the grating depth and number of gratings. The optimized design of a grating for coupling light into the waveguide that is to be placed into the PEEM sample chamber is the second task addressed in this thesis. 


\section{Photoemission Electron Microscopy (PEEM)}

Photoemission electron microscopy (PEEM) is a technique of forming images through collecting electrons emitted from sample surface by means of photoelectric effect. The spatial resolution in PEEM is thus limited by electron optics. As outlined in Ch. 1, PEEM can be used for probing the light propagation and interaction near a solid surface with spatial resolution below the light-optical diffraction limit [10].

The resolution in PEEM is determined by the electron de Broglie wavelength and optical aberrations. In our microscope a spatial resolution of $5 \mathrm{~nm}$ has been demonstrated [35]. Photoemission is an ultrafast process and the time resolution in PEEM is therefore also largely determined by the optical time resolution. While pulses of $\sim 80 \mathrm{fs}$ are used in our microscope, the pulse delay is reproducible and accurate down to $\sim 100$ as. Thus, the time resolution in the movie that is described in this thesis is of that order. The photoelectrons are usually generated within $\sim 10 \mathrm{~nm}$ of the sample surface containing ample surface information. Hence it is predominantly the intensity distribution on the sample surface that is imaged using PEEM [11,12].

\subsection{Theory and working principle of PEEM:}

The governing equation of photoemission process described by Einstein is formulated as,

$$
E_{k}=\hbar \omega-\varphi
$$

An electron can be emitted from a solid surface when it acquires sufficient kinetic energy $E_{k}$ by absorbing a photon with energy $\hbar \omega$. This process requires the photon energy to 
exceed the work function $\varphi$ that represents the minimum energy required for electron to escape the metal.

In contrast to the one-photon photoemission process described by equation (2.1), the multi-photon non-linear photoemission process does not require the photon energy to be higher than the work function for photoelectron yield. The electron can still be emitted through overcoming the work function by absorbing n photons quasi-simultaneously. The multiphoton photoemission process thus can be expressed as,

$$
E_{k}=n \hbar \omega-\varphi
$$

Here $\mathrm{n}$ can be any number of photons and their total energy must exceed the work function.

While a single-photon PEEM typically requires ultraviolet or x-ray sources to overcome the work function of many materials, a multiphoton PEEM image can be achieved by lower excitation energy provided by visible or infrared light sources. However, the photoemission yield is typically much lower in multi-photon emission.

The spatial distribution of photoelectron yield creates contrast for PEEM. For single-photon PE the photoemission yield $Y_{P E}$ is proportional to the light intensity which is proportional to the square of the electric field. Combining these two relations,

$$
\begin{array}{r}
Y_{P E} \propto|I| \\
\text { and } I \propto|\boldsymbol{E}|^{2}
\end{array}
$$




$$
\text { we get, } Y_{P E} \propto|\boldsymbol{E}|^{2}
$$

For n-photon photoemission process the photoemission yield is proportional to the intensity to the nth power, thus

$$
Y_{P E} \propto|\boldsymbol{E}|^{2 n}
$$

Hence for the multiphoton process, small variation in the intensity results in significantly more increase in photoemission yield than for the single photon process. Therefore, in multiphoton PEEM the contrast is more pronounced owing to its sensitivity to the variation of local photon density.

In PEEM the photoelectrons emitted from the sample are collected and accelerated through a series of lenses for magnification, apertures to limit the angular spread, deflectors to guide the electron beam through the optical column and a hyperbolic electron mirror for aberration correction. The electron beam is finally projected on a phosphor screen where it creates an image that is finally captured by a CCD camera equipped with a macro-objective. 


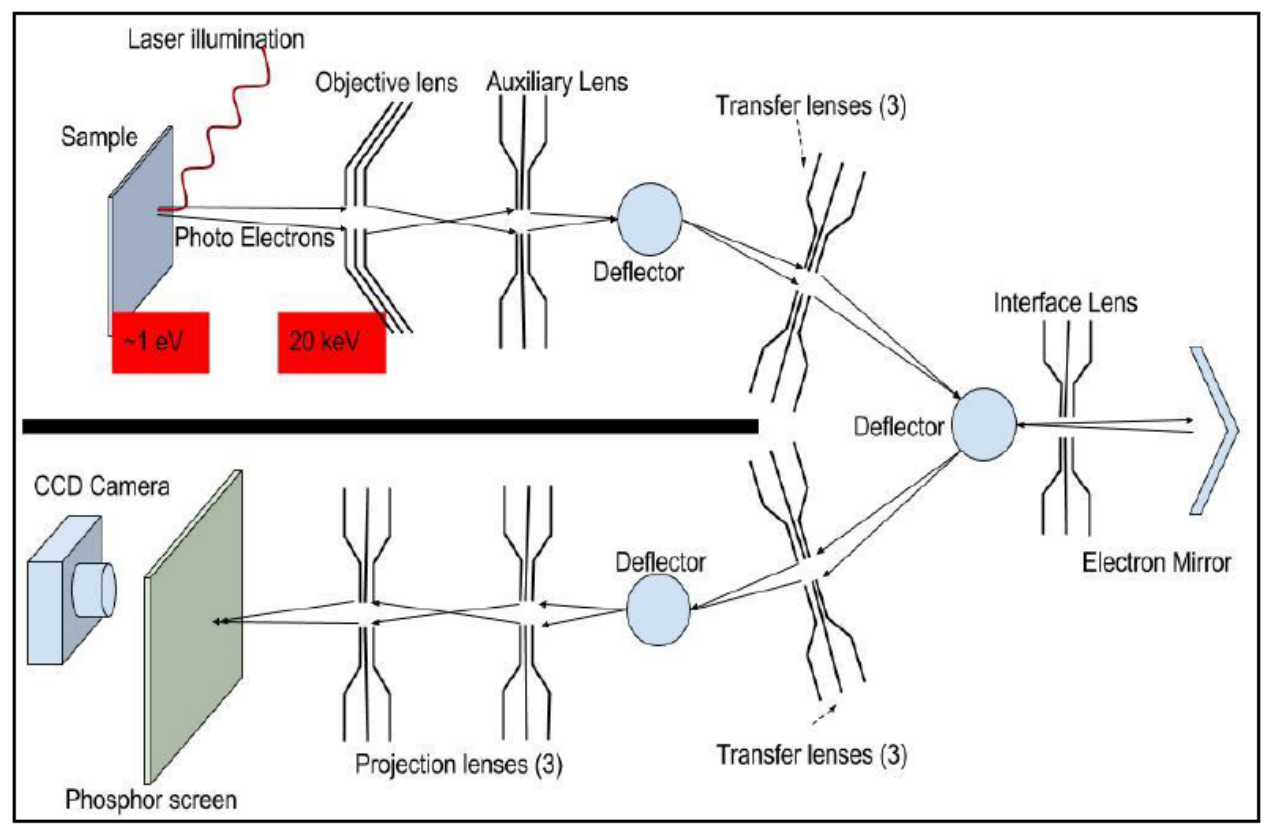

Figure 2. 2: Schematic diagram of PEEM. [15]

\subsection{Sample setup and experiment using PEEM:}

For this work the waveguide sample is placed in the microscope and illuminated by a pulsed pump beam to excite a mode. This guided mode propagates through the waveguide and interferes with a probe beam. The probe beam is coherent with the pump beam, as it is obtained from the pump beam in a beam splitter, and it can be delayed in a delay station. The interference pattern between the two beams provides a stationary intensity distribution that yields a spatially varying photoelectron distribution. This distribution is imaged using PEEM. Series of images are taken as the delay between the probe beam with respect to the pump beam is progressively increased. The images taken at different delays thus show a progressing interference pattern position. As the two partial beams interfere at right angles, the imaged interference pattern has the same 
wavelength and direction as the propagating mode squared. Furthermore, when a short laser is pulse is used, the length and amplitude distribution of the imaged interference pattern corresponds closely to that of the travelling mode pulse squared. Images taken with different delay times of the probing beam thus provide a direct observation of the travelling mode pulse, its temporal decay, scattering, decoherence and other properties. In other words, in compiling images taken with different delay between the two partial beams we effectively obtain a movie of the wave propagation through the waveguide. In this thesis we treat the case for continuous, not pulsed laser excitation, mostly because of computational limitations. Nonetheless, much of the insight obtained here can be used for the case of pulsed excitation that is actually realized in the PEEM set-up.

Our lab has a home-built aberration corrected PEEM system [13]. For sample illumination, the microscope uses a Spectra-Physics Mai Tai Ti: Sapphire laser with 60 fs pulse duration at a $100 \mathrm{MHz}$ repetition rate along with a second harmonic generator. This second harmonic generator converts the fundamental infrared pulses provided by the laser to wavelength of range 390- $415 \mathrm{~nm}$ range. For this work, $410 \mathrm{~nm}$ wavelength was considered as the source beam.

The PEEM setup allows the light beam incident on the sample at an angle $60^{\circ}$. This oblique beam act as the pump beam exciting a mode in the waveguide. Normal incidence can be achieved using a mirror below the sample [14]. A beam splitter is used to split the original beam coming from the source into a pump and a probe beam. The 
probe beam delay is produced by rotating a glass slide in the path of the beam.

\section{PEEM objective}

Probe beam Pump beam
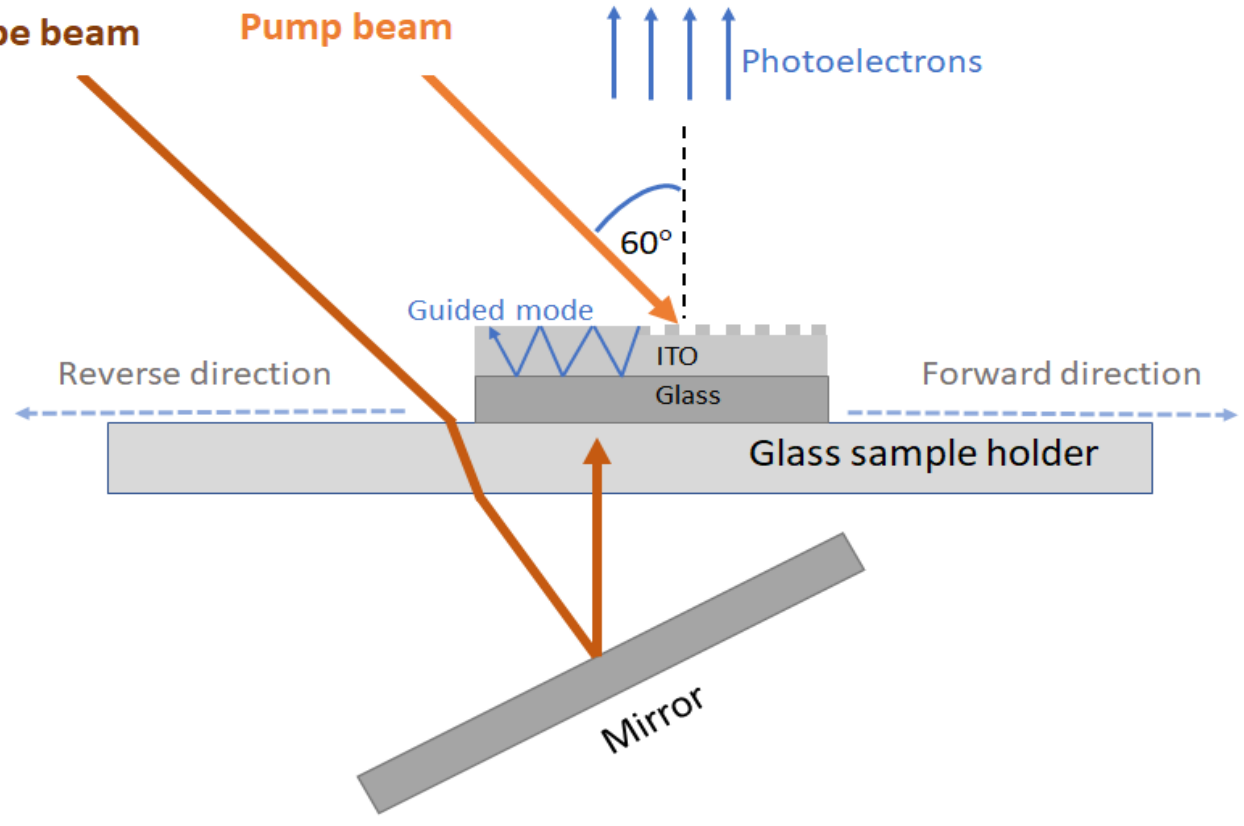

Figure 2.2: Schematic diagram of PEEM sample chamber containing the waveguide illuminated by the pump and the probe beam. [15]

In this thesis we simulate the light-optical portion of the experiment. We will discuss the properties of the interference pattern and demonstrate how the interference pattern progresses in space as the delay of the probe beam increases. We also discuss the presence of a stationary diffraction patterns in the vicinity of the moving interference pattern which complicates the analysis. To optimize the experiment, we also calculate an optimized optical grating for coupling the pump beam efficiently into the waveguide. The waveguide is a dielectric film that consists of a $180 \mathrm{~nm}$ thick ITO layer on a $200 \mathrm{~nm}$ thick glass substrate embedded in vacuum. A linear grating is scribed into the ITO using 
conventional FIB sculpting. The refractive index of ITO is $2.14 \pm 0.01$ at $410 \mathrm{~nm}$ wavelength. [15]. As the $410 \mathrm{~nm}$ pump beam wavelength corresponds to a photon energy of $3.02 \mathrm{eV}$ not enough to overcome the ITO work function $4.5 \mathrm{eV}$, a nonlinear 2-photon photoemission process takes place in the PEEM.

In the following we present first a general description of the waveguide, then in chapter 4 discuss the grating optimization. In chapter 5 we introduce the simulation approach and subsequently in chapter 6 present the simulation results for the interference pattern as well as its interpretation. Conclusions will be presented in chapter 7.

3. Coupled mode analysis for waveguides 
The waveguides serve as connections between the optical components of the integrated circuits. By confining light in optoelectronic devices, waveguides can provide long distance light signal transmission and channel it into desired direction. In this chapter, the structure of a basic waveguide, condition for the light to be confined within the waveguide and electric field distribution of the confined light within a dielectric waveguide is discussed.

\subsection{Waveguide structure:}

Optical waveguides consist of three layers - the substrate, the guiding layer, and the superstrate. The refractive index of the guiding layer is higher than that of the substrate and the superstrate so that light remain confined within the layer by means of the total internal reflection and propagates through interference

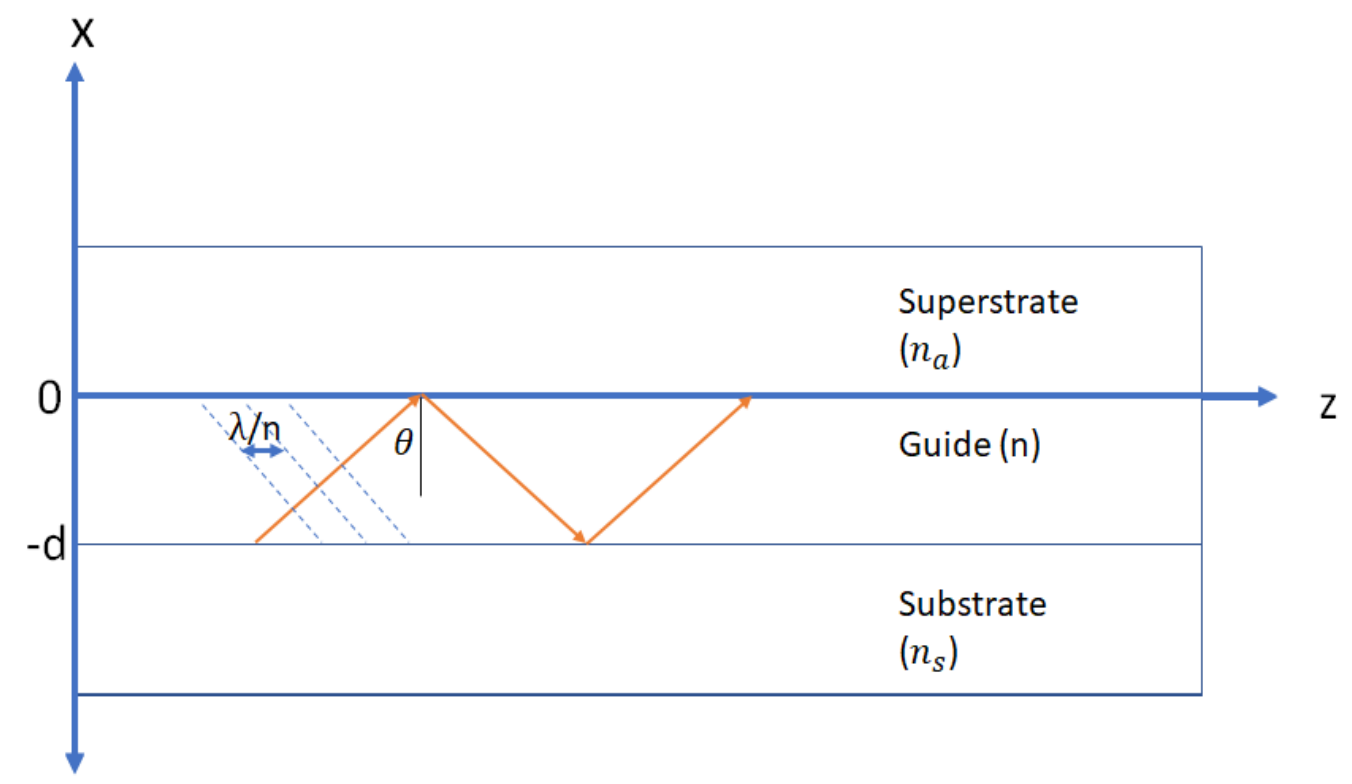

Figure 3. 5: Schematic representation of planar waveguide. 


\subsection{Guided modes (condition for light confinement):}

We consider light propagating in the $\mathrm{z}$ direction within a waveguide. The waveguide consists of a superstrate, the waveguide layer and a substrate medium. These have refractive indices $n_{a}, n$ and $n_{s}$ respectively. The thickness of the guide layer is $d$. We determine the dispersion relation for light in the waveguide by using the ray model. When the light propagates within the guiding layer and is incident on the guidingsubstrate and guiding- superstrate interface at angles greater than the corresponding critical angles, If $\theta_{\mathrm{c} 1}$ and $\theta_{\mathrm{c} 2}$, total internal reflection take place. The critical angles associated are given as, $\theta_{c 1}=\sin ^{-1} \frac{n_{a}}{n}$ and $\theta_{c 2}=\sin ^{-1} \frac{n_{s}}{n}$. Hence, total internal reflection at both interfaces take place when $\theta>\theta_{c 2}>\theta_{c 1}$ condition is satisfied with $\theta$ being the angle of incidence.
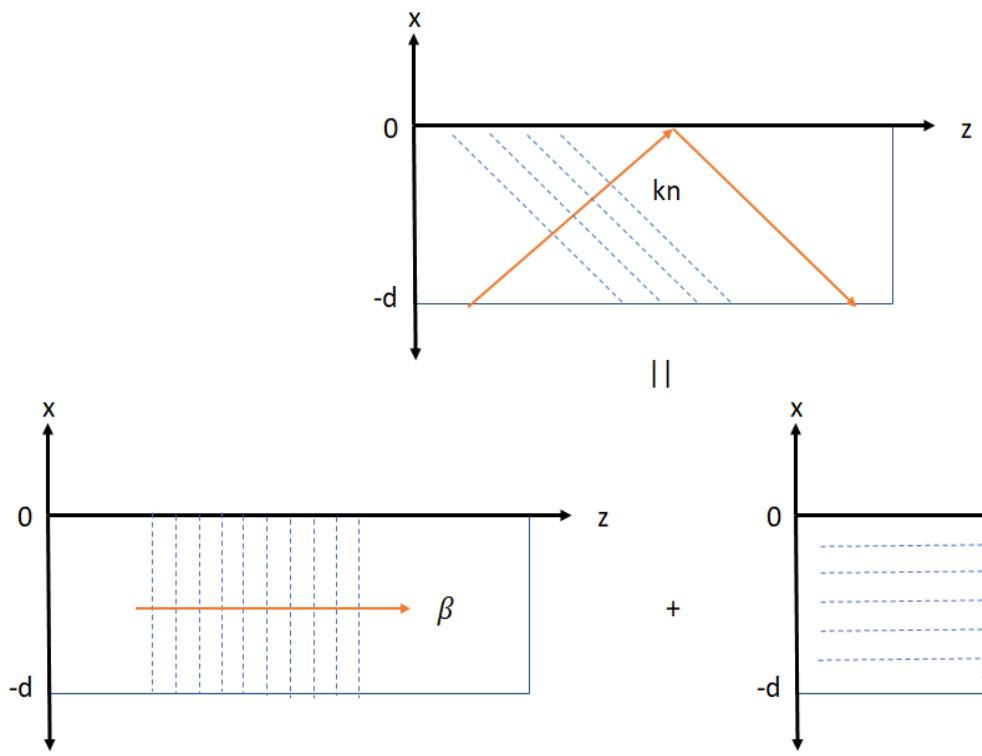

II

Figure 3.6: Wave propagation in the waveguide. 
This "zig zag" wave trapped within the guide by means of the total internal reflection can be considered as the combination of the orthogonal components travelling in the longitudinal $\mathrm{z}$ direction with propagation constant $\beta(=\mathrm{kn} \sin \theta)$ and the transverse $\mathrm{x}$ direction with propagation constant $\mathrm{h}(=\mathrm{kn} \cos \theta)$. The transverse component reflecting back and forth between the interfaces interferes with itself. The guided mode can exist only when this interference is constructive and thus the transverse resonance condition is satisfied. For this reason, to retain light propagation within the guide layer through constructive interference the total phase mismatch arising from the total internal reflection as well as the optical path difference should be a multiple of $2 \pi$ which can be expressed as following, [18]

$$
\Delta \phi-\Phi_{\mathrm{n}, \mathrm{n}_{\mathrm{a}}}-\Phi_{\mathrm{n}, \mathrm{n}_{\mathrm{s}}}=2 \mathrm{~m} \pi
$$

$\Delta \phi$ is the phase mismatch due to the optical path difference between two points and can be written as:

$$
\Delta \phi=2 \mathrm{ndk} \cos \theta=\frac{4 \pi}{\lambda} \mathrm{nd} \cos \theta
$$

$\Phi_{\mathrm{n}, \mathrm{n}_{\mathrm{a}}}$ and $\Phi_{\mathrm{n}, \mathrm{n}_{\mathrm{s}}}$ are the phase mismatch due to total internal reflection between the interface of cover region and the waveguide and between the substrate and the waveguide region respectively.

Equation (3.1) can be written as,

$$
2 \mathrm{knd} \cos \theta_{\mathrm{m}}=\Phi_{\mathrm{n}, \mathrm{n}_{\mathrm{a}}}+\Phi_{\mathrm{n}, \mathrm{n}_{\mathrm{s}}}+2 \mathrm{~m} \pi
$$




$$
\begin{aligned}
& 2 \mathrm{kd} \sqrt{\left(\mathrm{n}^{2}-\mathrm{N}_{\mathrm{m}}^{2}\right)}=\Phi_{\mathrm{n}, \mathrm{n}_{\mathrm{a}}}+\Phi_{\mathrm{n}, \mathrm{n}_{\mathrm{s}}}+2 \mathrm{~m} \pi \\
& \text { Where, } \mathrm{N}_{\mathrm{m}}=\mathrm{n} \sin \theta_{\mathrm{m}}
\end{aligned}
$$

Equation (3.4) is called the guided mode dispersion equation and indicates light propagating within a medium of a refractive index $n \sin \theta_{m}$ called the effective index $N_{m}$. Since m can only have integer values, equation (3.3) imposes only certain discrete values of $\theta_{\mathrm{m}}$ that can satisfy transverse resonance condition resulting in discrete values of the propagation constant $\beta_{\mathrm{m}}$ corresponding to individual guided modes.

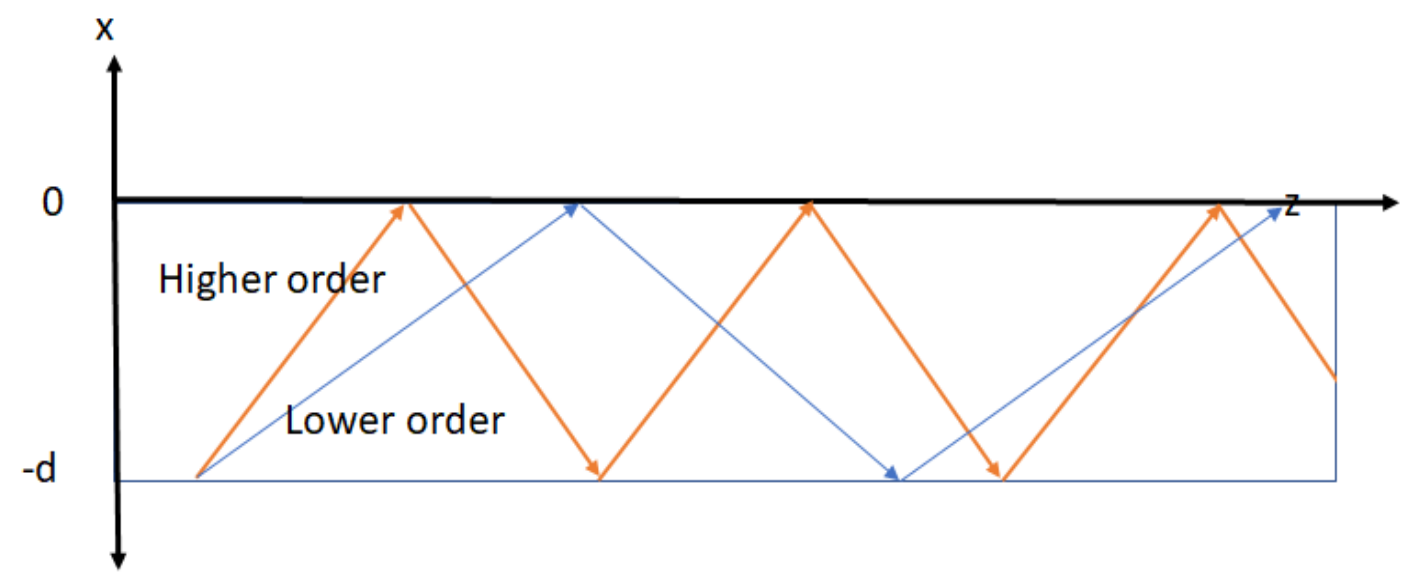

Figure 3. 7: Discrete modes with different $m$ values within the waveguide.

The phase mismatch due to the total internal reflection at the interfaces can be written as,

$$
\Phi_{\left(\mathrm{n}, \mathrm{n}_{\mathrm{j}}\right)}=\arctan \left[\left(\frac{\mathrm{n}}{\mathrm{n}_{\mathrm{j}}}\right)^{2 \rho} \sqrt{\frac{\left(\mathrm{N}_{\mathrm{m}}^{2}-\mathrm{n}_{\mathrm{j}}^{2}\right)}{\left(\mathrm{n}^{2}-\mathrm{N}_{\mathrm{m}}^{2}\right)}}\right]
$$

Where, $\rho=0$ for TE polarization and $\rho=1$ for TM polarization. 
Since the phase shifts due to the total internal reflection $\Phi_{n, n_{a}}$ and $\Phi_{n, n_{s}}$ for a given incident angle are dependent on polarization, the TE and TM modes have different solutions for the transverse resonant condition resulting in different effective indices for the same mode number $\mathrm{m}$.

\subsection{Electric field distribution}

In order to study the electric field distribution of the guided modes we need to find solution for Maxwell's equations that satisfy boundary conditions imposed by the waveguide structure. The electromagnetic wave within all three mediums of the waveguide structure is given by the following Maxwell's equations,

$$
\begin{aligned}
& \boldsymbol{\nabla} \times \mathbf{E}=-\mu_{0} \frac{\partial \mathbf{H}}{\partial \mathrm{t}} \\
& \boldsymbol{\nabla} \times \mathbf{H}=-\varepsilon_{0} \mathrm{n}^{2} \frac{\partial \mathbf{E}}{\partial \mathrm{t}} \\
& \boldsymbol{\nabla} . \mathbf{E}=0 \\
& \boldsymbol{\nabla} . \mathbf{H}=0 \\
& \mathbf{B}=\mu_{0} \mathbf{H} \\
& \mathbf{D}=\varepsilon \mathbf{E}
\end{aligned}
$$

For any TE mode of a planar waveguide, $\mathrm{E}_{\mathrm{z}}$ vanishes and according to the relations of the transverse components to the longitudinal components $\mathrm{E}_{\mathrm{x}}=\mathrm{H}_{\mathrm{y}}=0$ because $\frac{\partial \mathrm{H}_{\mathrm{z}}}{\partial \mathrm{y}}=0$ 
due to the y axis independence of index profile. So, the only non-vanishing field components are $\mathrm{H}_{\mathrm{x}}$, $\mathrm{E}_{\mathrm{y}}$ and $\mathrm{H}_{\mathrm{z}}$.

For transverse electric field we thus can write following from the Maxwell's equations,

$$
\begin{gathered}
\frac{\partial^{2} \mathrm{E}_{\mathrm{y}}}{\partial \mathrm{x}^{2}}+\left(\mathrm{k}^{2} \mathrm{n}^{2}-\beta^{2}\right) \mathrm{E}_{\mathrm{y}}=0 \\
\mathrm{H}_{\mathrm{x}}=-\frac{\beta}{\omega \mu_{0}} \mathrm{E}_{\mathrm{y}} \\
\mathrm{H}_{\mathrm{Z}}=-\frac{\beta}{\mathrm{i} \omega \mu_{0}} \frac{\partial \mathrm{E}_{\mathrm{y}}}{\partial \mathrm{x}}
\end{gathered}
$$

The Helmholtz equation (3.14) can be written for each medium as:

$$
\text { For air medium } n_{a}, \quad \frac{\partial^{2} E_{y}}{\partial x^{2}}-q^{2} E_{y}=0 \quad \text { where, } q^{2}=\beta^{2}-k^{2} n_{a}^{2}
$$

For waveguide medium

$$
\frac{\partial^{2} E_{y}}{\partial x^{2}}+h^{2} E_{y}=0 \quad \text { where, }
$$

n,

$$
\mathrm{h}^{2}=\mathrm{k}^{2} \mathrm{n}^{2}-\beta^{2}
$$

For substrate medium $n_{s}, \quad \frac{\partial^{2} E_{y}}{\partial x^{2}}-p^{2} E_{y}=0 \quad$ where, $\quad p^{2}=\beta^{2}-k^{2} n_{s}^{2}$

Here, $\beta$ is the longitudinal component of the propagation constant of the waveguide medium and $\beta=\mathrm{kN}_{\mathrm{m}}=\mathrm{kn} \sin \theta$ 
Now, for $\mathrm{h}^{2} \geq 0$ we get the sinusoidal solutions in the waveguide medium and for $\mathrm{q}^{2} \geq$ 0 and $\mathrm{p}^{2} \geq 0$ we get the evanescent waves in the air and the substrate medium which is expressed as following:

$$
\begin{array}{ll}
E_{y}(x)=C e^{-q x} & \text { for } \quad 0 \leq x \leq \infty \\
E_{y}(x)=A \cos (h x)+B \sin (h x) & \text { for }-d \leq x \leq 0 \\
E_{y}(x)=D e^{p(x+d)} & \text { for }-\infty \leq x \leq-d
\end{array}
$$

Where, $\mathrm{kn} \geq \beta \geq \mathrm{kn}_{\mathrm{s}} \geq \mathrm{kn}_{\mathrm{a}}$

Where the constants A, B, C and D is determined by means of imposing boundary conditions requiring continuity of $\mathrm{E}_{\mathrm{y}}$ given as following at the air-waveguide and substrate- waveguide interface,

$$
\begin{gathered}
\left.E_{y}\right|_{n}=\left.E_{y}\right|_{n_{a}} \\
\left.\frac{d E_{y}}{\partial x}\right|_{n}=\left.\frac{d E_{y}}{\partial x}\right|_{n_{a}} \\
\left.E_{y}\right|_{n}=\left.E_{y}\right|_{n_{s}} \\
\left.\frac{d E_{y}}{\partial x}\right|_{n}=\left.\frac{d E_{y}}{\partial x}\right|_{n_{s}}
\end{gathered}
$$

By applying these boundary conditions, we can write equations [3.20] [3.21] [3.22] as:

$$
\mathrm{E}_{\mathrm{y}}(\mathrm{x})=\mathrm{C} \text { for } \quad 0 \leq \mathrm{x} \leq \infty
$$




$$
\begin{array}{ll}
E_{y}(x)=C\left[\cos (h x)-\frac{q}{h} \sin (h x)\right] & \text { for } \quad-d \leq x \leq 0 \\
E_{y}(x)=C\left[\cos (h d)+\frac{q}{h} \sin (h d)\right] e^{p(x+d)} & \text { for } \quad-\infty \leq x \leq-d
\end{array}
$$

The electric field distribution corresponding to each guided mode can be determined from above equations. The propagation constant $\mathrm{C}$ and $\beta$ can be calculated by using derivative continuity of the electromagnetic field in the guide which leads to:

$$
\tan (h d)=\frac{p+q}{h-\frac{p q}{h}}
$$

Which yields the dispersion relation upon simplification:

$$
\mathrm{hd}=\tan ^{-1} \frac{\mathrm{q}}{\mathrm{h}}+\tan ^{-1} \frac{\mathrm{p}}{\mathrm{h}}+\mathrm{m} \pi
$$

Same dispersion relation can also be acquired by solving for $\mathbf{H}$.

Solution of the above eigenvalue equation can be obtained graphically, the example of which is provided in the following figure: 


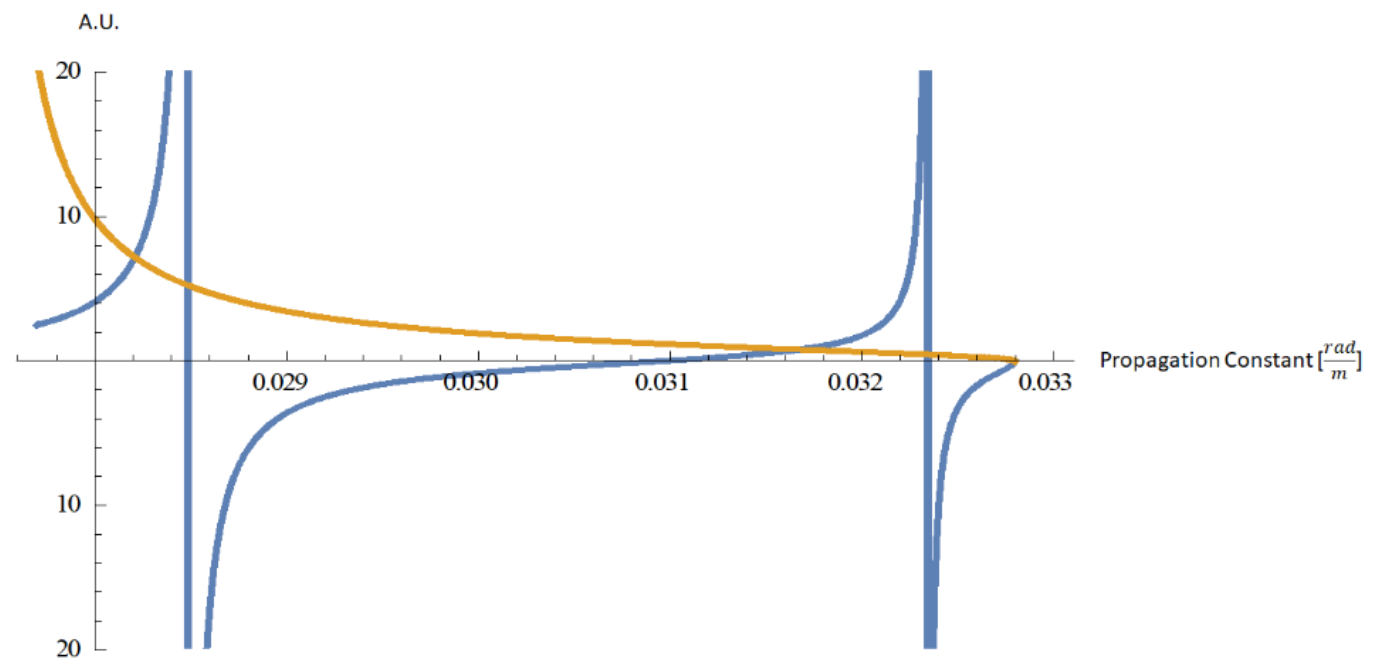

Figure 3.4: The graphical solution for the propagation constant of an ITO waveguide of 290nm thickness and 2.14 refractive index with air superstrate and glass substrate with refractive index 1.5 at a wavelength of $410 \mathrm{~nm}$. The propagation constants are determined from the non-asymptotic intersections.

From the graphical solution, our sample ITO waveguide which is $180 \mathrm{~nm}$ is found to permit a single mode of effective index of 1.885 for TM polarization. 


\section{Grating coupler}

In chapter 3 we have discussed how the guided modes propagate being confined within the waveguides. While the waveguides serve as the interconnect between the optical components of an integrated circuits, the guided modes cannot be launched into the waveguide by illuminating it. Exciting a guided mode into the waveguide requires phase matching elements such as diffraction gratings, prisms, lenses. The coupling methods using prism and lens are not compatible with the size of the integrated optical circuits and causes difficulty in alignment. The diffraction grating on the other hand is a compact and efficient coupler that can be easily introduced into the device structure providing better alignment compared to the facet coupling using lens and prism. [33][34] Optimization of the structural design of grating couplers are critical for efficient coupling of desired modes within a specific waveguide in a certain direction. In this chapter the theory of diffraction grating and the optimization of gratings for efficient coupling is discussed.

\subsection{Theory of Diffraction Grating}

\subsubsection{Scalar wave theory}

Let us consider, an electromagnetic plane wave propagating in the xz plane, incident from a homogeneous dielectric medium of refractive index $n_{1}$ upon the grating that diffracts it into the refracted and transmitted orders.

The interaction of a grating with an incident beam in diffracting it into transmitted or reflected orders is given by the Floquet condition as, 


$$
\mathbf{k}_{\mathrm{q}}=\mathbf{k}_{0}-\mathrm{qK}
$$

Where $\mathbf{K}$ is the grating constant with magnitude $|\mathbf{K}|=\frac{2 \pi}{\Lambda}$ and directs toward the periodicity $\Lambda, q$ represents diffracted orders $0, \pm 1, \pm 2 \ldots ., \mathbf{k}_{0}$ represents wave vector of undiffracted $0^{\text {th }}$ order and $\mathbf{k}_{\mathrm{q}}$ is the wave vector of the qth diffracted order. The scalar representation of above equation along $\mathrm{x}$ axis is given as,

$$
\begin{aligned}
& \frac{2 \pi n_{t}}{\lambda} \sin \theta_{q}=\frac{2 \pi n_{t}}{\lambda} \sin \theta_{0}-\frac{2 \pi q}{\Lambda} \\
& \text { The Snell's law: } n_{1} \sin \theta_{1} \\
&=n_{3} \sin \theta_{0}
\end{aligned}
$$

Where $n_{1}$ and $n_{3}$ are index of refraction of incident and transmitted medium respectively, $\lambda$ is the wavelength of incident beam, $\theta_{0}$ is the angle of undiffracted $0^{\text {th }}$ order, $\theta_{1}$ is the angle of the incident beam and $\theta_{q}$ is the angle of qth diffracted order. These angles are directional quantities and is measured from the surface normal. The sign of clockwise angles are taken to be negative and that of the counterclockwise angles are taken to be positive.

Incorporating the Snell's law with equation (4.3) Error! Reference source not found.Error! Reference source not found.Error! Reference source not found.Error! Reference source not found.Error! Reference source not found.Error! Reference source not found. Error! Reference source not found.Error! Reference source not found.Error! Reference source not found.Error! Reference source not found.Error! Reference source not found.Error! Reference source not found.we get, 


$$
\frac{2 \pi n_{3}}{\lambda} \sin \theta_{q}=\frac{2 \pi n_{1}}{\lambda} \sin \theta_{1}-\frac{2 \pi q}{\Lambda}
$$

Which yields the diffraction equation [19],

$$
n_{3} \sin \theta_{q}=n_{1} \sin \theta_{1}-\frac{q \lambda}{\Lambda}
$$

From (4.5) we see that the allowed values of $q$ are limited by the value of $\sin \theta_{q}$ as

$$
\begin{gathered}
-1 \leq \frac{n_{1}}{n_{3}} \sin \theta_{i}-\frac{q \lambda}{\Lambda n_{3}} \leq 1 \\
\text { or, } \frac{\Lambda}{\lambda}\left(n_{3}+n_{1} \sin \theta\right) \leq q \leq-\frac{\Lambda}{\lambda}\left(n_{1} \sin \theta-n_{3}\right)
\end{gathered}
$$




\subsubsection{Electromagnetic theory}

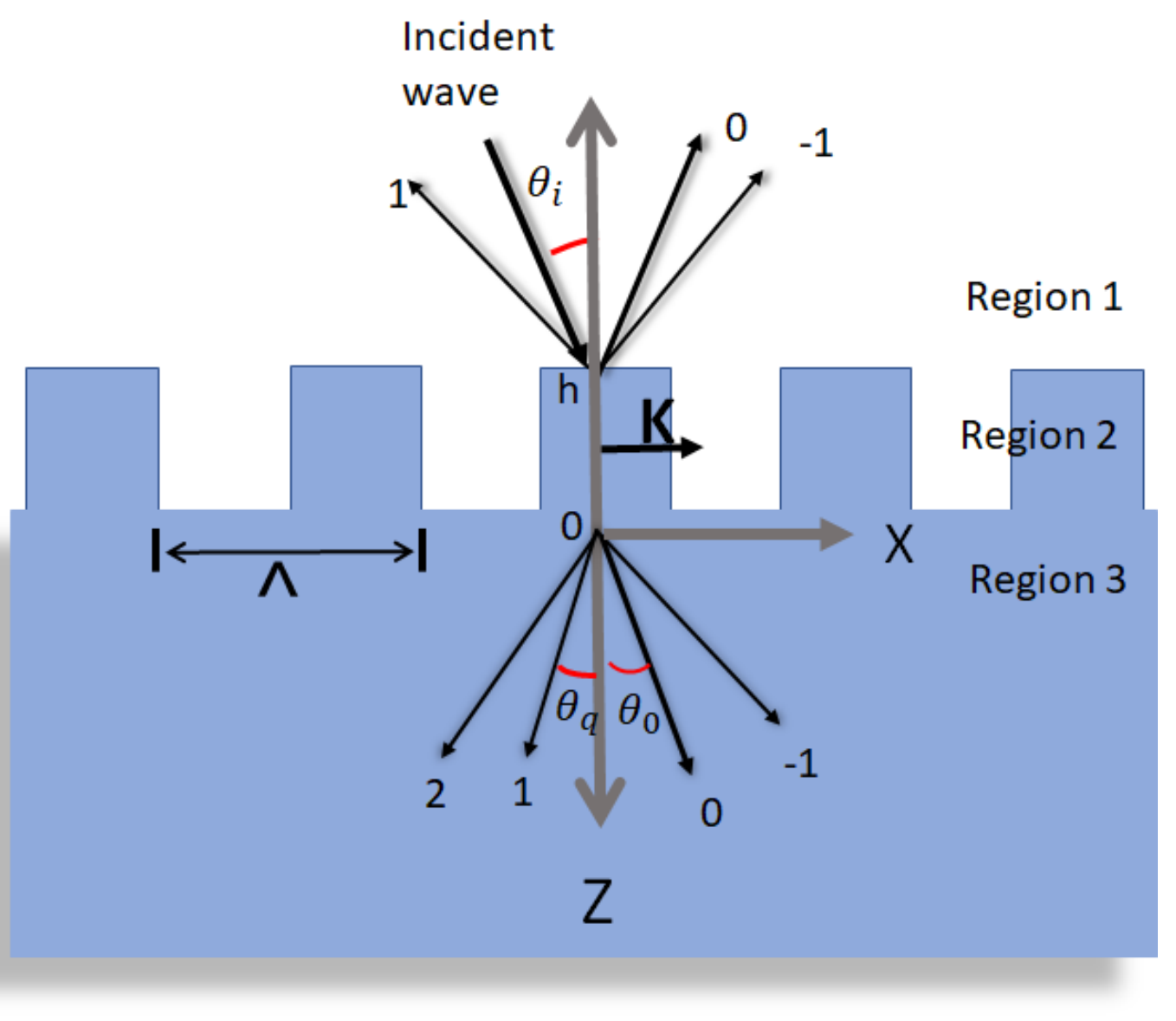

Figure 4. 8: transmitted and reflected orders of a binary diffraction grating.

Let us consider the electromagnetic plane wave propagating in region 1 which is a homogeneous dielectric medium before it is incident on the grating (region 2) which is the modulated region of thickness $\mathrm{h}$ and period $\Lambda$. Upon diffracted by the grating the plane wave gets partially reflected to the region 1 and partially transmitted to the homogeneous dielectric region 3.

The solution of the grating problem can be obtained by finding an electromagnetic field satisfying Maxwell's equation as well as the boundary conditions everywhere. 
The Maxwell's equations can be written as following where due to the y axis independence of the index profile, the y derivatives vanish. [20]

$$
\begin{gathered}
i \omega B_{x}(x, z)=-\frac{\partial}{\partial z} E_{y}(x, z) \\
i \omega B_{z}(x, z)=-\frac{\partial}{\partial x} E_{y}(x, z) \\
\frac{\partial}{\partial z} H_{x}(x, z)-\frac{\partial}{\partial x} H_{z}(x, z)=J_{y}(x, z)-i \omega D_{y}(x, z) \\
J_{x}(x, z)-i \omega D_{x}(x, z)=-\frac{\partial}{\partial z} H_{y}(x, z) \\
J_{z}(x, z)-i \omega D_{z}(x, z)=\frac{\partial}{\partial x} H_{y}(x, z) \\
\frac{\partial}{\partial z} E_{x}(x, z)-\frac{\partial}{\partial x} E_{z}(x, z)=i \omega B_{y}(x, z)
\end{gathered}
$$

These equations lead to the Helmholtz equation given as:

$$
\frac{\partial^{2}}{\partial x^{2}} U(x, z)+\frac{\partial^{2}}{\partial z^{2}} U(x, z)+k^{2} \hat{\varepsilon}_{r}(x, z) U(x, z)=0
$$

$\hat{\varepsilon}_{r}(x, z)$ is the complex relative permittivity and $[\hat{n}(x, z)]^{2}$ is the complex refractive index.

$$
\hat{\varepsilon}_{r}(x, z)=[\hat{n}(x, z)]^{2}=\frac{\varepsilon(x, z)}{\varepsilon_{0}}+\frac{i \sigma(x, z)}{\omega \varepsilon_{0}}
$$


Here, $\mathrm{U}=E_{y}$ for TE mode and $\mathrm{U}=H_{y}$ for $\mathrm{TM}$ mode. The total electric field can be written as $\mathrm{U}=U_{p}+U_{D}$ with $U_{p}$ being the incident field that vanishes for all regions except region 1 :

$$
U_{p}(x, z)=\exp \left[i k n_{1}(x \sin \theta+z \cos \theta)\right]
$$

$U_{D}$ is the diffracted field and is periodic with the grating period d such that, $U_{D}(x+$ $\Lambda, z)=\exp \left(i k_{0} \Lambda\right) U_{D}(x, z)$.

$$
U_{D}(x, z)=\sum_{q=-\infty}^{\infty} G_{q}(z) \exp \left(i k_{q} x\right)
$$

Where, $k_{q}=k n_{1} \sin \theta-\frac{2 \pi q}{\Lambda}$ which leads to the grating equation (4.5) and the periodic function $G(x, z)=U_{D}(x, z) \exp \left(-i k_{0} x\right)$ is expressed in the form of Fourier series in $\mathrm{x}$ with Fourier coefficients $G_{q}(z)$.

Now by inserting equation (4.17) into the Helmholtz equation (4.14) and solving it we get the plane wave representation of the diffracted field in region 1 and 3.

In region 3 we get the transmitted field given as:

$$
U_{T}(x, z)=\sum_{q=-\infty}^{\infty} T_{q} \exp \left\{i\left[k_{q} x+t_{q}(z-h)\right]\right\}
$$

With the sign convention,

$$
t_{q}=\left[\left(k n_{3}\right)^{2}-k_{q}^{2}\right]^{\frac{1}{2}} \text { for }\left|k_{q}\right| \leq k n_{3}
$$




$$
t_{q}=i\left[k_{q}^{2}-\left(k n_{3}\right)^{2}\right]^{\frac{1}{2}} \text { for }\left|k_{q}\right|>k n_{3}
$$

For the real values of $t_{q}$, we get the homogeneous plane waves which are the propagating diffraction orders of the grating and for the imaginary values of $t_{q}$ we get the evanescent waves decaying exponentially as $z \rightarrow \infty$.

Similarly, in region 1 we get the reflected field given as,

$$
U_{R}(x, z)=\sum_{q=-\infty}^{\infty} R_{q} \exp \left\{i\left[k_{q} x-r_{q} z\right]\right\}
$$

With the sign convention,

$$
\begin{gathered}
r_{q}=\left[\left(k n_{1}\right)^{2}-k_{q}^{2}\right]^{\frac{1}{2}} \text { for }\left|k_{q}\right| \leq k n_{1} \\
r_{q}=i\left[k_{q}^{2}-\left(k n_{1}\right)^{2}\right]^{\frac{1}{2}} \text { for }\left|k_{q}\right|>k n_{1}
\end{gathered}
$$

Where the plane waves are associated with the real $r_{q}$ and the evanescent waves are associated with imaginary $r_{q}$. The total electric field in region 1 is,

$$
\begin{aligned}
& U=U_{p}+U_{D}=\exp \left[i k n_{1}(x \sin \theta+z \cos \theta)\right]+ \\
& \sum_{q=-\infty}^{\infty} R_{q} \exp \left\{i\left[k_{q} x-r_{q} z\right]\right\}
\end{aligned}
$$

Finally, the electric field distribution in the modulation region 2 can be obtained from the Helmholtz equation by means of separation of variables,

$$
\begin{aligned}
& U(x, z)=\sum_{l=-\infty}^{\infty} \sum_{n=1}^{\infty} P_{l n} \exp \left(i k_{l} x\right)\left\{a_{n} \exp \left(i \gamma_{n} z\right)\right. \\
& \left.+b_{n} \exp \left(-i \gamma_{n}(z-h)\right)\right\}
\end{aligned}
$$


where $\exp \left( \pm i \gamma_{n} z\right)$ terms represent bouncing waves in the waveguide. and coefficients $P_{l n}$ depend on $\varepsilon(x, z)$.

We now have the exact representation of the electromagnetic field in all three regions and the parameters $T_{q}, R_{q}, a_{n}, b_{n}$ are determined by using electromagnetic boundary conditions.

\subsection{Optimized grating coupler design}

\subsubsection{Types of grating}

In general gratings can be of two types - amplitude grating and phase grating.

The amplitude gratings spatially modulate only the amplitude of the transmitted wave that is diffracted by its array of slits.

On the other hand, phase gratings spatially modulate the phase of the transmitted wave that is diffracted by its narrow ridges of periodically alternating indices of refraction. The phase gratings are generally of two types- surface relief gratings and volume gratings.

Surface relief gratings have alternating index of refraction by periodical change of its surface topography. Usually, the refractive index alters between that of the substrate on which the grating is situated and the covering region which is in most times air. The surface relief grating with periodic thin rectangular ridges or grooves are called binary grating and are known for being able to manufacture easily. Other two types of surface 
relief gratings are the blazed gratings with triangular ridges and sinusoidal grating with sinusoidal surface profile.
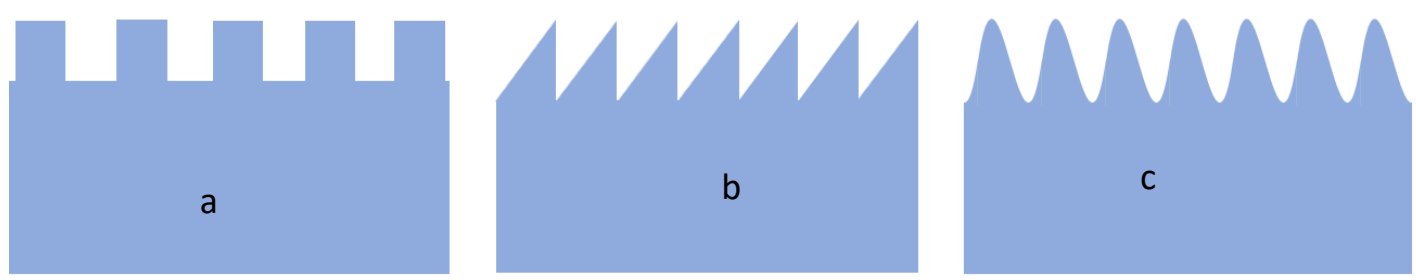

Figure 4.9: (a) binary grating, (b) blazed grating and (c) sinusoidal grating.

In contrast to surface relief grating, the volume phase gratings do not have any open grooves but have a plane surface topography. These types of gratings consist of an optically thick film with periodically alternating refractive index that is sandwiched between two substrates.

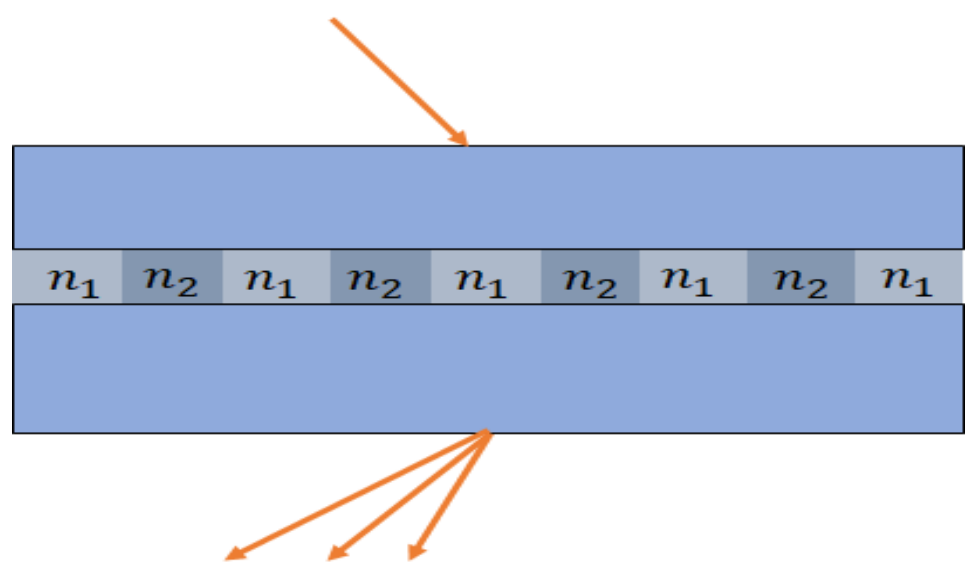

Figure 4.10: Volume Grating. 


\subsubsection{Optimizing parameters}

Diffraction gratings are very useful means of coupling modes into the waveguides as they are capable of channeling significant portion of the incident beam power into the desired diffracted orders at certain angles that determine their corresponding effective indices. The grating profile plays a great role in the coupling process. While the grating periodicity determines which of the diffracted orders is coupled for a given medium and incident light, the coupling efficiency however depends on the grating depth, shape and number of grating elements.

Diffraction gratings serve as guided mode couplers by means of effective refractive index matching. The effective refractive index of the guided mode is defined as the ratio of the corresponding propagation constant to the propagation constant of free space. Similarly, the effective refractive index of a diffracted order is defined as the ratio of the tangential component of its propagation constant to the propagation constant in the free space. Depending on the effective refractive index of the diffracted orders, three types of coupling can take place- waveguide coupling, free space coupling and substrate coupling. 


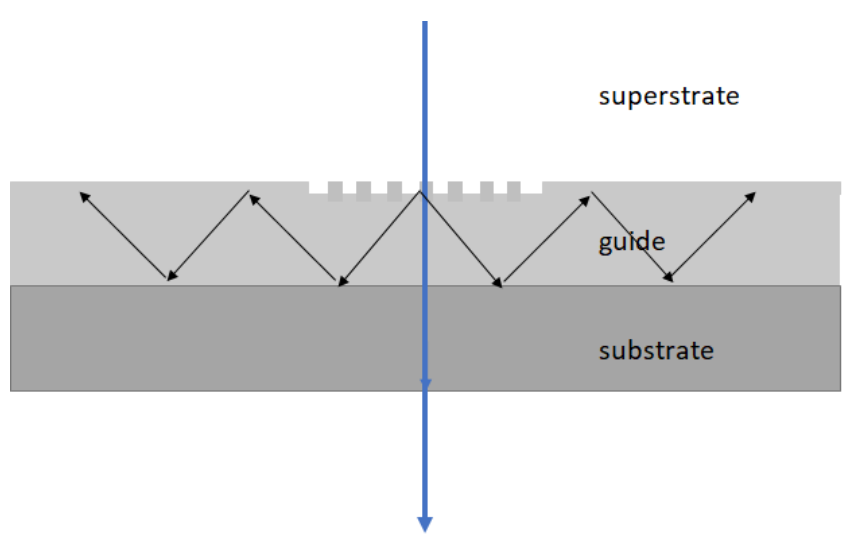

Figure 11.4: waveguide coupling with normally incident beam.

When the effective index of one of the guided modes match with the effective index of a diffracted order, the light incident on the grating gets successfully coupled into the waveguide. In this case the value of the effective index of the waveguide coupled order is lower than the refractive index of the waveguide, but higher than that of the substrate. If the effective indices of the diffractive orders are higher than refractive index of the waveguide, all the orders get cut off and only the undiffracted $0^{\text {th }}$ order beams exist.

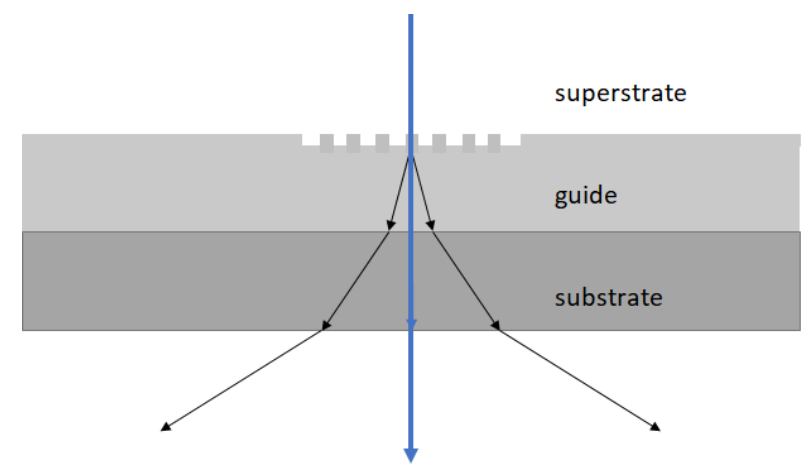

Figure 4.12: Free space coupling 
On the other hand, free space coupling takes place when the effective indices of the diffracted orders are lower than the refractive index of the free space and the diffracted orders exist in all three regions.

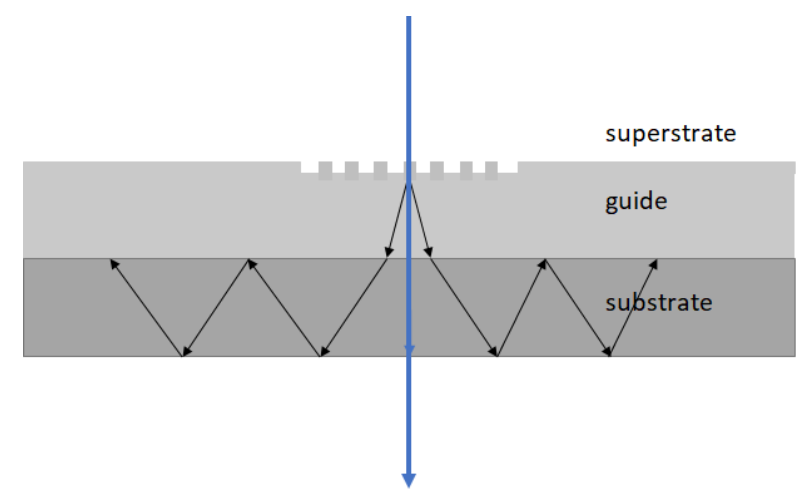

Figure 4.6: Substrate coupling.

Finally, substrate coupling occurs when the effective indices of the transmitted orders are less than the refractive index of the substrate, but greater than that of the free space. In this case the transmitted order remain confined within the substrate by means of total internal reflection.

The effective index of $q^{\text {th }}$ diffracted order:

$$
\eta_{\text {eff }}^{q}=\frac{k_{q x}}{k_{\text {free }}}=\frac{\frac{2 \pi n_{3}}{\lambda} \sin \theta_{q}}{\frac{2 \pi}{\lambda}}=n \sin \theta_{q}
$$

Combining above with the Floquet condition $\left(n \sin \theta_{q}=n \sin \theta_{1}-\frac{q \lambda}{\Lambda}\right)$ we get,

$$
\eta_{e f f}^{q}=n_{3} \sin \theta_{q}=n_{1} \sin \theta_{1}-\frac{q \lambda}{\Lambda}
$$


Hence, the $q^{\text {th }}$ transmitted order will be coupled into the waveguide if $\eta_{\text {eff }}^{q}$ is adjusted by means of the incident angle, grating period or the wavelength of the incident beam to match with the effective index of the modes allowed within the waveguide.

Since the PEEM setup only allows $60^{\circ}$ angle of incidence and the incident wavelength is chosen to be $410 \mathrm{~nm}$, we have to adjust the grating period to couple the desired mode into the waveguide.

When the beam is incident normally on the grating, by choosing a suitable $\Lambda$, both $\pm \mathrm{q}$ orders can be coupled simultaneously. For example, our sample ITO waveguide is $180 \mathrm{~nm}$ thick and permits a single mode of effective indices of 1.885 for TM polarization. If $\Lambda=149 \mathrm{~nm}$ is chosen for normal incidence, both +1 and -1 orders are coupled. But for an oblique incidence such as for $\theta_{i}=60^{\circ}$, the -1 order is coupled in when $\Lambda=402 \mathrm{~nm}$ while the +1 order is cut off. When $\Lambda=149 \mathrm{~nm}$, the +1 order is coupled in and the -1 order is cut off. This is the preferred coupling in our experiments. 


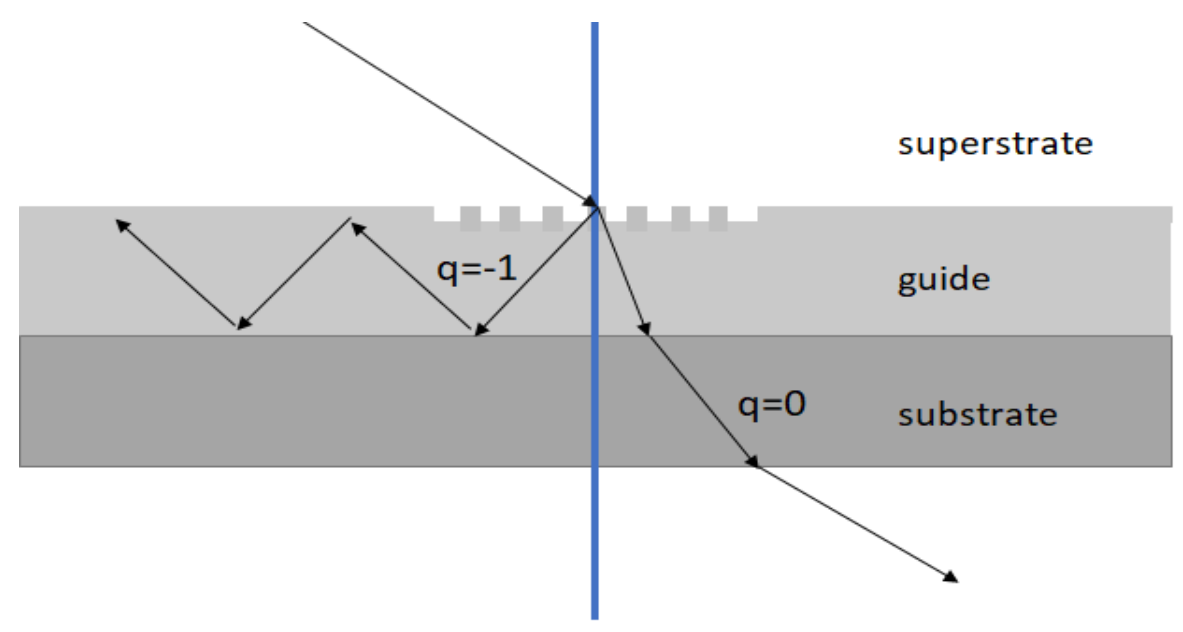

Figure 4.13: Waveguide coupling for oblique incident beam.

\subsubsection{Coupled power}

While coupling of the desired mode is done by choosing the grating period accordingly, what fraction of the incident power is coupled into the waveguide is tuned by the grating depth and the number of grating elements. In order to find the coupled power, the time averaged power flow expressed below is determined through the cross section of the waveguide,

$$
<\boldsymbol{S}(\boldsymbol{r}) \geq \lim _{T \rightarrow \infty} \frac{1}{2 T} \int_{-T}^{T} \boldsymbol{E}(\boldsymbol{r}, t) \times \boldsymbol{H}(\boldsymbol{r}, t) d t=\frac{1}{2} \operatorname{Re}\{\boldsymbol{E}(\boldsymbol{r}) \times \boldsymbol{H} *(\boldsymbol{r})\}
$$




\section{Simulation}

In this work, the optimized grating parameters and the interference movie were obtained by numerical analysis. For the interference movie, the PEEM images of the interference pattern on the sample surface for varying delays between the pump and the probe beam serve as snapshots. To simulate a snapshot, an image of the time averaged real portion of the electric field distribution of the interference pattern across the sample is produced. This image of electric field distribution is equivalent to the PEEM image as the photoemission yield creating contrast in the PEEM image is proportional to this field. Numerical analysis is also used for determining coupled power across the waveguide to optimize grating parameters for efficient coupling. Since the complex structure of grating couplers requires advanced numerical techniques, Finite Element Method is used as a method of simulation.

This chapter illustrates simulation methods used for obtaining results for this thesis.

\subsection{Finite Element Method:}

The finite element method is a numerical procedure for approximating solutions to boundary value problems governed by the differential equations and appropriate boundary conditions.

This method is facilitated by means of discretization where the entire continuous domain is divided into a number of subdomains known as mesh elements. The primary unknow quantity such as the potential or the electric field is represented as a weighted 
sum of basis functions which are assigned to the node or edge of each mesh elements. The differential equations are then written in weak forms to obtain local matrices for each meshing elements. These local matrices are assembled into global matrix for the overall domain and then is solved.

The first step of a finite element analysis is discretization through mesh generation. The mesh elements can be triangular or rectangular elements in $2 \mathrm{D}$ or tetrahedrons or hexahedrons in 3D. To conform perfectly to the complex geometry mesh generation procedure depends on some basic requirements. The elements should not overlap or have gaps between them. The vertex of an element can only be at the vertex of another element and cannot be inside of another element. An optimized mesh element also should not have narrow elements or elements having small inner angle, as it increases the solution error. Hence it is desired to make the elements closer to equilateral for triangular mesh. [21, p 94]

Also, smaller elements give more accurate result but requires increased memory space and computing time. Which is resolved by refining mesh locally by generating smaller mesh where the features are small or fields vary abruptly and larger mesh elsewhere. By doing so as few elements as possible are used to obtain accurate solution. Optimized meshing generation procedure is complex and it exploits elaborated theory out of scope of this thesis. [26,27]

After discretization, the next step in FEM is to expand the unknown solution assigned to the nods or the edges of an element into a set of basis function called the 
"shape function". The solution inside the element is then interpolated using the set of scalar or vector shape function.

A simple form of a scalar shape function in $2 \mathrm{D}$ domain is the shape function of a linear triangular Lagrange element or a nodal element. Then the expression for the unknown solution in any element e with node I can be written as, [24, p 31]

$$
\varphi^{e}=\sum_{j=1}^{3} N_{j}^{e} \varphi_{j}^{e}
$$

Here, $\varphi_{j}^{e}$ is the value of $\varphi$ for node $\mathrm{j}$ and $N_{j}^{e}$ is the shape function for node $\mathrm{j}$. A triangular element has 3 nods (vertices) and the shape functions only depend on the coordinates of these nodes.

Then continuity of the interpolated solutions across the edges between two elements are given as the following relation,

$$
N_{l}^{e}\left(x_{k}^{e}, y_{k}^{e}\right)= \begin{cases}1 & l=k \\ 0 & l \neq k\end{cases}
$$

Where $\left(x_{k}^{e}, y_{k}^{e}\right)$ is the coordinates of the nodes of element e. That is for each node of an element the corresponding shape function equals to one and it vanishes for other nodes of that element.

The scalar shape functions are suitable for approximating scalar field such as potential. However, using scalar shape function in discretizing vector field such as electric field results in spurious solution. A vector shape function is more suitable in that 
case. In this approach the tangential component of the vector field is assigned at each edge of the element and the field elsewhere is interpolated using the vector basis function. [28 -32] One type of vector shape function or basis function is Whitney 1- form basis function.[22,23] This type of basis function for a triangular element e corresponding to the edge connecting the nodes $l$ and $\mathrm{k}$ can be written as,

$$
\boldsymbol{N}_{l k}^{e}(\boldsymbol{r})=\left[N_{l}^{e} \nabla N_{k}^{e}-N_{k}^{e} \nabla N_{l}^{e}\right] l_{l k}^{e} \quad l<k
$$

This function corresponding to a particular edge of the element is a nonzero constant while it vanishes for other edges for the same element.
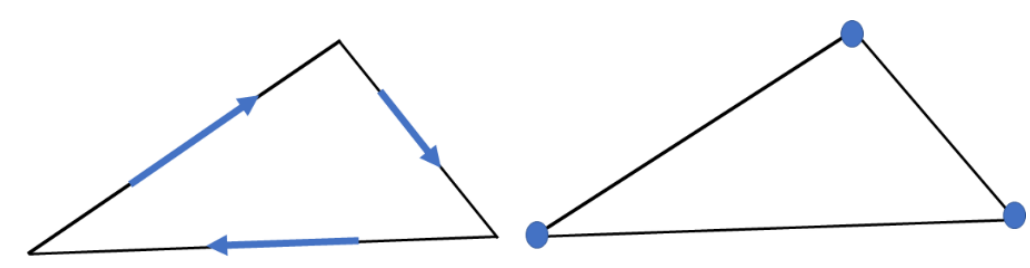

Figure 5. 6: edge and node triangular elements.

The electric field in element e with edges denoted by 1,2,3 can be approximated as,

$$
\boldsymbol{E}^{e}=\boldsymbol{N}_{12}^{e} E_{12}^{e}+\boldsymbol{N}_{13}^{e} E_{13}^{e}+\boldsymbol{N}_{23}^{e} E_{23}^{e}
$$

After interpolating the electric field for each element, the electric field over the entire domain can be approximated as,

$$
E=\sum_{j=1}^{N} \boldsymbol{N}_{j} E_{j}
$$


Where $\mathrm{N}$ is the total number of edges in the entire domain, $E_{j}$ is the tangential component of electric field on the jth edge and $\boldsymbol{N}_{j}$ is the corresponding vector basis function.

Now after replacing the entire domain by appropriate meshing and expressing the unknown solution of the differential equation associated with each meshing element in terms of shape function, we obtain the weak formulation of the differential equation expressing the physical problem of interest.

The differential equation governing the coupled guided mode propagation as well as the scattered wave propagation is the Helmholtz wave equation,

$$
\boldsymbol{\nabla} \times\left(\frac{1}{\mu} \boldsymbol{\nabla} \times \mathbf{E}\right)-k_{0}^{2} \varepsilon \boldsymbol{E}=\mathbf{0}
$$

Using $\mu=1$ and $\varepsilon=n^{2}$ the equation can be alternatively written as,

$$
\boldsymbol{\nabla} \times(\boldsymbol{\nabla} \times \mathbf{E})-k_{0}^{2} n^{2} \boldsymbol{E}=\mathbf{0}
$$

The weak formulation of the differential equation is derived using the weighted residual method or the variational method to obtain corresponding linear equation. For this work, the Galerkin's method is used which is a weighted residual method.

By writing equation (5.7) in terms of the basis function we have,

$$
\boldsymbol{\nabla} \times\left(\boldsymbol{\nabla} \times \sum_{j=1}^{N} \boldsymbol{N}_{j} E_{j}\right)-k_{0}^{2} n^{2} \sum_{j=1}^{N} \boldsymbol{N}_{j} E_{j}=0
$$


A suitable test function is then multiplied to the above equation. According to the Galerkin's method this test function is the same as the shape function.

$$
\boldsymbol{N}_{i} . \boldsymbol{\nabla} \times\left(\boldsymbol{\nabla} \times \sum_{j=1}^{N} \boldsymbol{N}_{i} \boldsymbol{N}_{j} E_{j}\right)-k_{0}^{2} n^{2} \boldsymbol{N}_{i} \cdot \sum_{j=1}^{N} \boldsymbol{N}_{i} \boldsymbol{N}_{j} E_{j}=0
$$

Then integrating both side over the problem domain $\Omega$ gives,

$$
\int \boldsymbol{N}_{i} \cdot \boldsymbol{\nabla} \times\left(\boldsymbol{\nabla} \times \sum_{j=1}^{N} \boldsymbol{N}_{j} E_{j}\right) d \Omega=k_{0}^{2} n^{2} \int \boldsymbol{N}_{i} \cdot \sum_{j=1}^{N} \boldsymbol{N}_{j} E_{j} d \Omega
$$

By using the following vector identity on the left side of the above equation,

$$
A .(\nabla \times \nabla \times B)=(\nabla \times A) .(\nabla \times B)-\nabla .(A \times \nabla \times B)
$$

We get,

$$
\begin{array}{r}
\int\left(\boldsymbol{\nabla} \times \boldsymbol{N}_{i}\right) \cdot\left(\boldsymbol{\nabla} \times \sum_{j=1}^{N} \boldsymbol{N}_{j} E_{j}\right) d \Omega \\
-\int \boldsymbol{\nabla} \cdot\left(\boldsymbol{N}_{i} \times \boldsymbol{\nabla} \times \sum_{j=1}^{N} \boldsymbol{N}_{j} E_{j}\right) d \Omega \\
\left.=k_{0}^{2} n^{2} \int \boldsymbol{N}_{i} \cdot \sum_{j=1}^{N} \boldsymbol{N}_{j} E_{j} d \Omega\right)
\end{array}
$$

Using Gauss's theorem yields,

$$
\begin{array}{r}
\begin{array}{r}
\sum_{j=1}^{N} E_{j} \int\left(\boldsymbol{\nabla} \times \boldsymbol{N}_{i}\right) \cdot\left(\boldsymbol{\nabla} \times \boldsymbol{N}_{j}\right) d \Omega- \\
-\sum_{j=1}^{N} E_{j} \oint_{S}\left(\boldsymbol{N}_{i} \times \boldsymbol{\nabla} \times \boldsymbol{N}_{j}\right) \cdot \boldsymbol{d} \boldsymbol{S} \\
=k_{0}^{2} n^{2} \sum_{j=1}^{N} E_{j} \int \boldsymbol{N}_{i} \cdot \boldsymbol{N}_{j} d \Omega
\end{array} \\
\begin{array}{r}
\sum_{j=1}^{N} E_{j}\left(\int\left(\boldsymbol{\nabla} \times \boldsymbol{N}_{i}\right) \cdot\left(\boldsymbol{\nabla} \times \boldsymbol{N}_{j}\right) d \Omega-\oint_{S}\left(\boldsymbol{N}_{i} \times \boldsymbol{\nabla} \times \boldsymbol{N}_{j}\right) \cdot \boldsymbol{d} \boldsymbol{S}-\right. \\
\left.k_{0}^{2} n^{2} \int \boldsymbol{N}_{i} \cdot \boldsymbol{N}_{j} d \Omega\right)=0
\end{array}
\end{array}
$$


This yields the following matrix equation,

$$
[Z][E]=0
$$

Where,

$$
\begin{aligned}
& Z_{i j}=\int\left(\boldsymbol{\nabla} \times \boldsymbol{N}_{i}\right) \cdot\left(\boldsymbol{\nabla} \times \boldsymbol{N}_{j}\right) d \Omega-\oint_{S}\left(\boldsymbol{N}_{i} \times \boldsymbol{\nabla} \times \boldsymbol{N}_{j}\right) \cdot \boldsymbol{d} \boldsymbol{S}- \\
& k_{0}^{2} n^{2} \int \boldsymbol{N}_{i} \cdot \boldsymbol{N}_{j} d \Omega
\end{aligned}
$$

The matrix equation has to be solved for $[\boldsymbol{E}]$ which is the vector unknown coefficients $\boldsymbol{E}_{j}=\left\{E_{1}, \ldots . E_{j}, \ldots . E_{N}\right\}$. once $[\boldsymbol{E}]$ is obtained, the field everywhere within the domain can be calculated using $E=\sum_{j=1}^{N} N_{j} E_{j}$.

The coupled power is determined in terms of the fields as the Pointing's vector is expressed as follows:

$$
\boldsymbol{S}=\frac{1}{2} \operatorname{Re}\{\boldsymbol{E}(r) \times \boldsymbol{H} *(r)\}
$$

Integrating The above equation over a surface element yields power flow through the surface.

$$
P=\int_{S} \boldsymbol{S} . d \boldsymbol{S}=\int_{S} \frac{1}{2} \operatorname{Re}\{\boldsymbol{E}(r) \times \boldsymbol{H} *(r) . d \boldsymbol{S}\}
$$

For $3 \mathrm{D}$ problems having symmetry along one axis, it can be reduced to a $2 \mathrm{D}$ problem. Hence the expression for power flow across a line corresponds to:

$$
\int_{S} \boldsymbol{S} . d \boldsymbol{S}=L \int_{c} \boldsymbol{S} . d \boldsymbol{l}
$$




\subsection{Simulation Model:}

The electromagnetic problem under consideration concerns a sample with features having dimensions comparable to the wavelength. Such smaller features require constructing finer meshing that leads to a numerous quantity of meshing elements. Fast solving wave equation for each of those elements requires a commercial software. The mediums considered here are homogeneous and the fields vary sinusoidally with time in a given frequency range. Hence the problem can be considered as frequency domain.

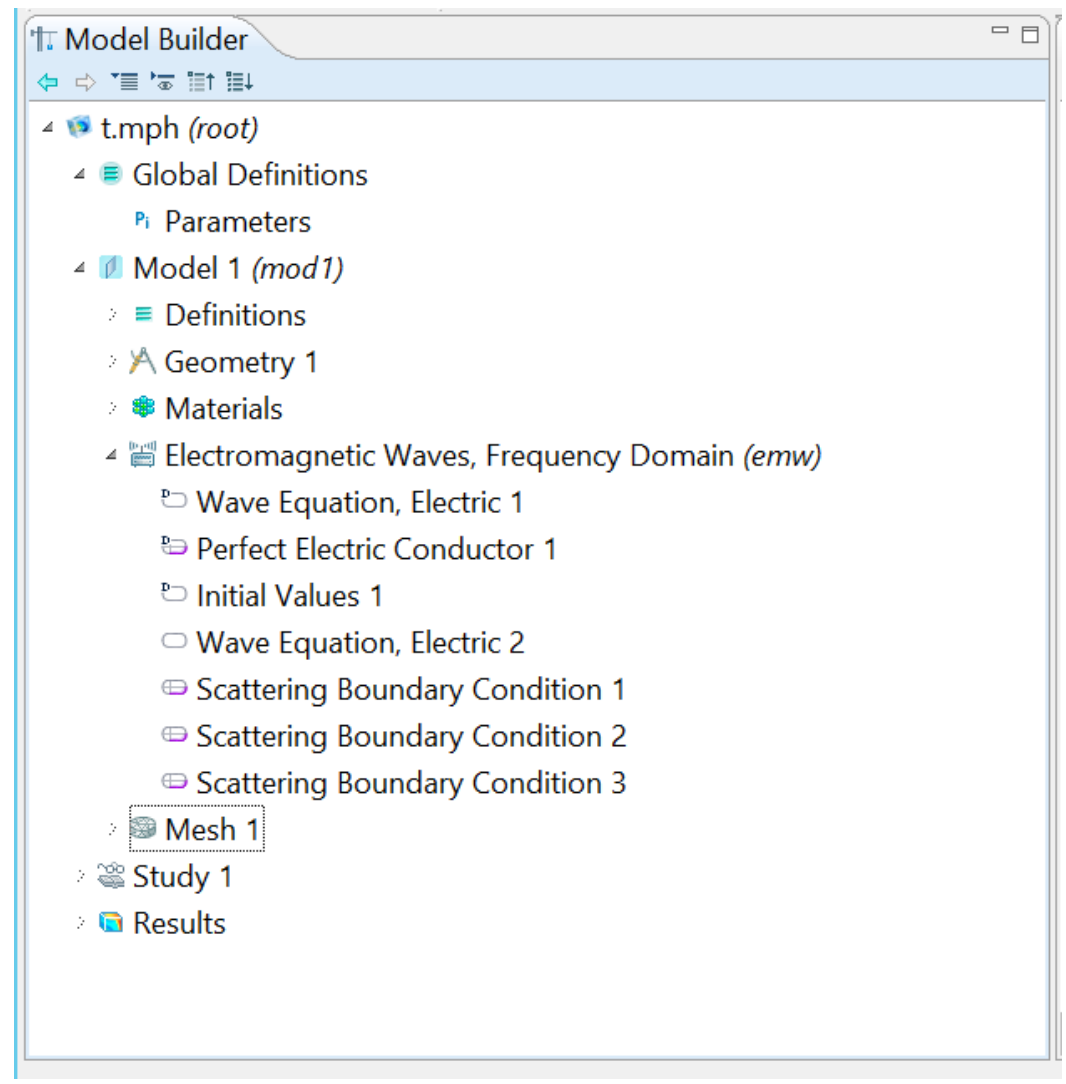

Figure 5. 7: COMSOL desktop environment.

COMSOL Multiphysics software is used for simulation in this work. It has a dedicated physics interface that uses finite element method to solve the Maxwell's 
equations in the frequency domain. The RF (radio frequency) module is used to access such modeling tools. Detail about COMSOL RF module is provided in the user's manual.[25] Selecting the appropriate physics interface and associated module is the first step before model building using COMSOL.

The geometry and physics of the model can be controlled using a parameter list. The globally defined parameters such as sample dimension can be used throughout the modeling process which makes it easier and less time consuming. The parametric sweep feature accelerates the model set up for finding efficient parameters. For example, to find the optimum grating width and size for efficient coupling, it is necessary to solve several variations of the same model by changing these parameters. Using parametric sweep offers an alternative to manually changing these parameters and re-solving each time. The phase delay between the pump and probe pulse were varied using the parametric sweep feature to create electric field image snap shots through a range.

After setting the parameters, they are used in various steps of creating the model geometry. Instead of building a 3D geometry, a cross section viewed in xz- plane is built. This is done because there is no variation along y axis. This $2 \mathrm{D}$ approximation takes less computational memory and yields faster result. After building the geometry, each part of the structure was assigned appropriate material from the built-in material library included in COMSOL that contains a large number of materials along with their properties.

The mesh was then created based on the geometry; coarse meshing was used for parts with larger features while finer meshing was created where the features were small. 
Free triangular mesh was used where the maximum element size was kept less than one fifth of the wavelength in the free space.

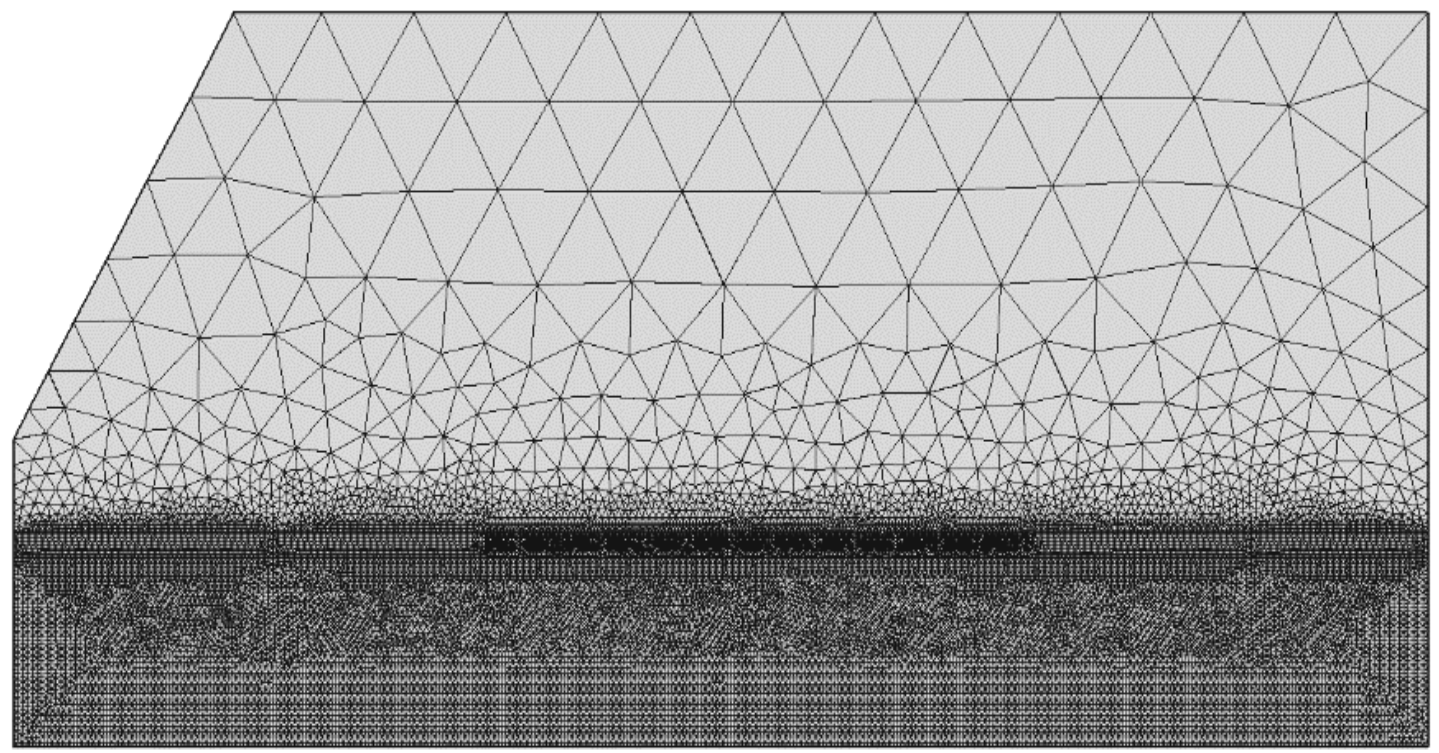

Figure 5. 8: Triangular meshing throughout the model.

In the following step a field distribution is created by using the Port boundary condition of the COMSOL RF module. This field distribution corresponds to the oblique pump beam incident on the ITO surface in air medium. This beam excites the guided mode in the ITO layer. For the interference movie model, an additional port is excited at the bottom of the substrate boundary that corresponds to the probe beam.

Computing the solution at this stage provides the electric field distribution image across the model 


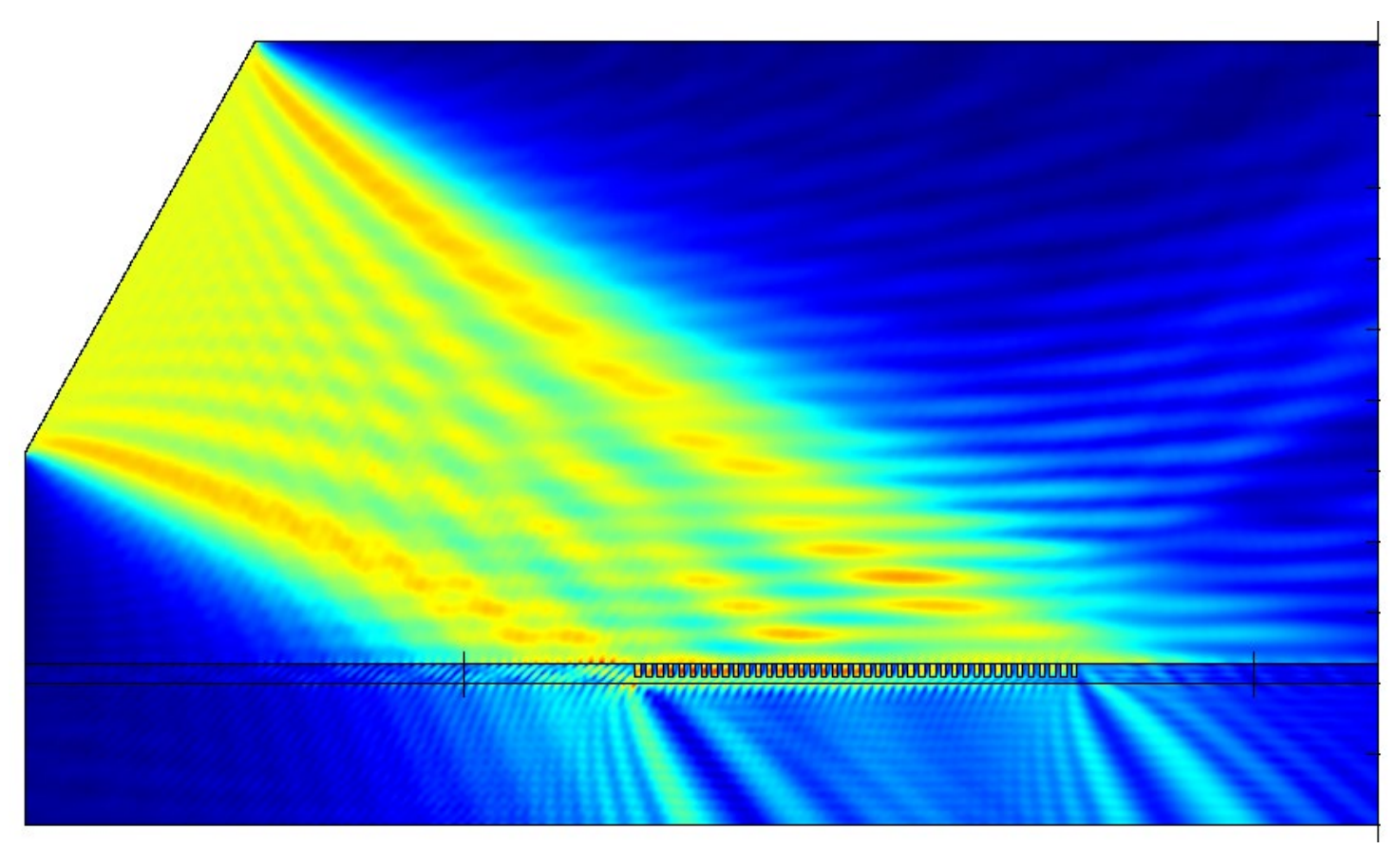

Figure 5. 9: Electric field distribution image result.

Next step involves postprocessing, where the coupled power is calculated and compared for optimization. By utilizing power sweep feature, the grating size and width is varied. The corresponding coupled power is then calculated using the line integration (5.19) across the red and blue line at the ITO cross section given in the image below. The blue line lies across the reverse direction while the red line lies in the forward direction. The goal here is to achieve maximum power flow in the reverse direction across the blue line and record the corresponding grating parameters to create the model for interference movie. 


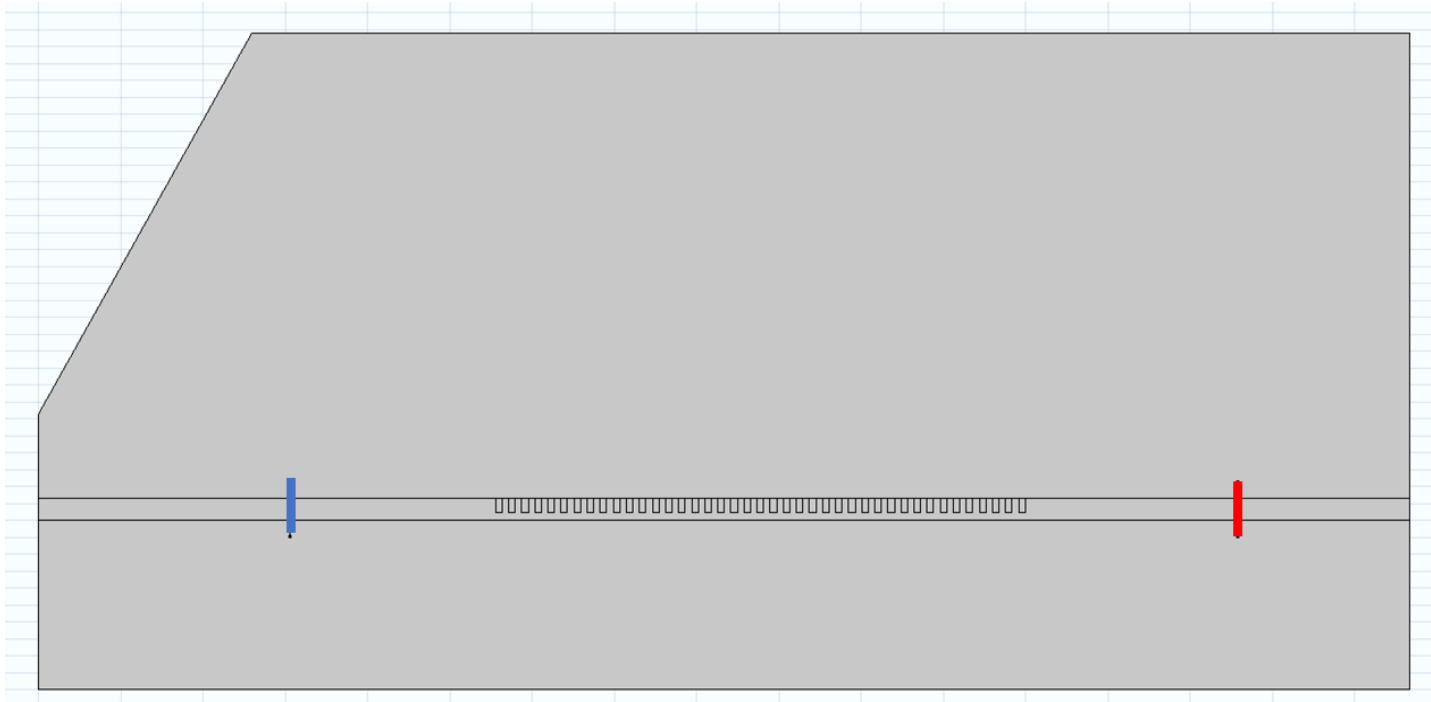

Figure 5. 10: Power flow calculation through the blue and red lines across ITO thickness

Using COMSOL, optimized grating parameters are determined for both binary and blazed grating by comparing corresponding coupled power of the guided mode. This optimization was done to acquire efficient coupling of light in the reverse direction for TM polarization.

These gratings are etched on $180 \mathrm{~nm}$ thick ITO structure sandwiched between a glass substrate and Aire superstrate. The optimized model obtained in this way is then used observe the interference pattern created by the guided mode and the probe pulse on the ITO surface. The line graph of the time averaged electric field intensity on the ITO surface is then extracted for varying delay between the probe beam and the beam exciting the mode. 


\section{Results}

We simulate the image of the time averaged electric field intensity distribution across the ITO sample. The total electromagnetic field $\mathbf{E}_{\text {tot }}$ at any position is the result of the summation of the fields corresponding to an arbitrary incident $\mathbf{E}_{\text {inc }}$ wave and the guided wave $\mathbf{E}_{\mathrm{m}}$, both at the same frequency.

$$
\mathbf{E}_{\text {tot }}=\mathbf{E}_{\text {inc }}+\mathbf{E}_{\mathrm{m}}
$$

The field amplitude of the incident wave and the guided mode at time $\mathrm{t}$ and position $y$ on the surface is $E_{\text {inc }}(y, t)=A e^{i(k y \sin \theta-\omega t)}$ and $\mathrm{E}_{\mathrm{m}}(y, t)=$ $B e^{i\left(-k y n_{e f f}-\omega t\right)}$ respectively. Here $\mathrm{k} \sin \theta$ is the magnitude of incident wave vector along $\mathrm{y}$ direction and $-\mathrm{k} n_{e f f}$ is the magnitude of the wave vector of the guided mode propagating along $-y$ direction. The corresponding intensity distribution on the surface will be, $I_{E M}=\left\|E_{t o t}\right\|^{2}$. The probability of $\mathrm{n}$ photon photoemission will be proportional to the nth power of the electromagnetic field intensity, $\mathrm{Y}_{\mathrm{n}, \mathrm{PE}}(\mathrm{t})=\mathrm{I}_{\mathrm{EM}}^{\mathrm{n}}=\left\|\mathrm{E}_{\mathrm{tot}}\right\|^{2 \mathrm{n}}$. Hence for a 2- photon photoemission process which is required for this study, the photoemission yield [12],

$$
\begin{aligned}
Y_{P E}(t)=I_{E M}^{2} & =\left\|E_{\text {tot }}\right\|^{4} \\
& =\left\|E_{\text {inc }}+E_{m}\right\|^{4} \\
& =\left\|A e^{i(k y \sin \theta-\omega t)}+B e^{i\left(-k y n_{e f f}-\omega t\right)}\right\|^{4}
\end{aligned}
$$


Here, $\quad\left\|E_{\text {tot }}\right\|^{2}=E_{\text {tot }} \times E_{\text {tot }}{ }^{*}$

$$
\begin{aligned}
= & {\left[A e^{i(k y \sin \theta-\omega t)}+B e^{i\left(-k y n_{e f f}-\omega t\right)}\right] \times\left[A e^{i(-k y \sin \theta+\omega t)}+\right.} \\
& \left.B e^{i\left(k y n_{e f f}+\omega t\right)}\right] \\
= & A^{2}+B^{2}+A B e^{i\left(k y \sin \theta+k y n_{e f f}\right)}+A B e^{-i\left(k y \sin \theta+k y n_{e f f}\right)} \\
= & A^{2}+B^{2}+A B\left[e^{i\left(k y \sin \theta+k y n_{e f f}\right)}+e^{-i\left(k y \sin \theta+k y n_{e f f}\right)}\right]
\end{aligned}
$$

And

$$
\begin{aligned}
\left\|E_{\text {tot }}\right\|^{4}=\left[A^{2}+\right. & \left.B^{2}+A B e^{i\left(k y \sin \theta+k y n_{e f f}\right)}+A B e^{-i\left(k y \sin \theta+k y n_{e f f}\right)}\right]^{2} \\
=A^{4} & +B^{4}+2 A^{2} B^{2}\left[e^{2 k y i\left(\sin \theta+n_{e f f}\right)}+e^{-2 k y i\left(\sin \theta+n_{e f f}\right)}\right] \\
& +2 A^{2} B^{2}+2 A B^{3}\left[e^{k y i\left(\sin \theta+n_{e f f}\right)}+e^{-k y i\left(\sin \theta+n_{e f f}\right)}\right] \\
& +2 A^{3} B\left[e^{k y i\left(\sin \theta+n_{e f f}\right)}+e^{-k y i\left(\sin \theta+n_{e f f}\right)}\right]
\end{aligned}
$$

Hence the intensity distribution for both single photon $n=1$ and 2-photon photoemission process $n=2$ will be independent of time yielding a stationary interference pattern.

For the 2- photon photoemission process the photoemission yield over pulse length of m cycles would also be independent of time. 


$$
\begin{aligned}
Y_{P E}= & \int_{0}^{m T}\left\|E_{t o t}\right\|^{4} d t \\
= & \int_{0}^{m T}\left\{A^{4}+B^{4}+4 A^{2} B^{2} \cos ^{2}\left[2 k y\left(\sin \theta+n_{e f f}\right)\right]+2 A^{2} B^{2}+\right. \\
& \left.4 A B^{3} \cos \left[\mathrm{yk}\left(\sin \theta+n_{e f f}\right)\right]+4 A^{3} B \cos \left[\mathrm{yk}\left(\sin \theta+n_{e f f}\right)\right]\right\} d t \\
= & m T\left\{A^{4}+B^{4}+4 A^{2} B^{2} \cos ^{2}\left[2 k y\left(\sin \theta+n_{e f f}\right)\right]+2 A^{2} B^{2}+\right. \\
& \left.4 A B^{3} \cos \left[\mathrm{yk}\left(\sin \theta+n_{e f f}\right)\right]+4 A^{3} B \cos \left[\mathrm{yk}\left(\sin \theta+n_{e f f}\right)\right]\right\}
\end{aligned}
$$

As a result, we observe two different wave numbers $k_{\text {int, } 1}=k\left(n_{\text {eff }}+\sin \theta\right)$ and $k_{\text {int, } 2}=2 k\left(n_{\text {eff }}+\sin \theta\right)$ in the interference pattern for 2-photon photoemission process and only one wave number $k_{\text {int }, 1}=k\left(n_{\text {eff }}+\sin \theta\right)$ for single photon photoemission process. The signal corresponding to $k_{i n t, 1}$ will have intensity proportional to $A^{3} B$ and $A B^{3}$ with $A^{3} B$ being more intense as the amplitude $\mathrm{A}$ of the incident beam will be greater than the amplitude B of the guided mode. The signal corresponding to $k_{\text {int, } 2}$ will have intensity proportional to $A^{2} B^{2}$.

The corresponding spacing between the interference maxima will be,

$$
\mathrm{d}_{1}=\frac{2 \pi}{\mathrm{k}_{\mathrm{int}, 1}}=\frac{2 \pi}{\mathrm{k}\left(\mathrm{n}_{\mathrm{eff}}+\sin \theta\right)} \quad \text { and } \quad \mathrm{d}_{2}=\frac{2 \pi}{\mathrm{k}_{\mathrm{int}, 2}}=\frac{2 \pi}{2 \mathrm{k}\left(\mathrm{n}_{\mathrm{eff}}+\sin \theta\right)}
$$

Since for present study the beam interfering with the mode is incident normally, $\sin \theta=$ $\sin 0=0$ and,

$$
\mathrm{d}_{1}=\frac{2 \pi}{\mathrm{k}_{\mathrm{int}, 1}}=\frac{2 \pi}{\mathrm{kn}_{\mathrm{eff}}} \quad \text { and } \quad \mathrm{d}_{2}=\frac{2 \pi}{\mathrm{k}_{\mathrm{int}, 2}}=\frac{2 \pi}{2 \mathrm{kn}_{\mathrm{eff}}}
$$


We extract a line graph of the field distribution from the simulated normalized field distribution image of the ITO surface for analysis. This line graph represents the change of the electric field with respect to the distance from the grating coupler. The actual image captured by the 2 - photon photoemission process will show the intensity distribution that is proportional to the $4^{\text {th }}$ power of this simulated electric field distribution values.

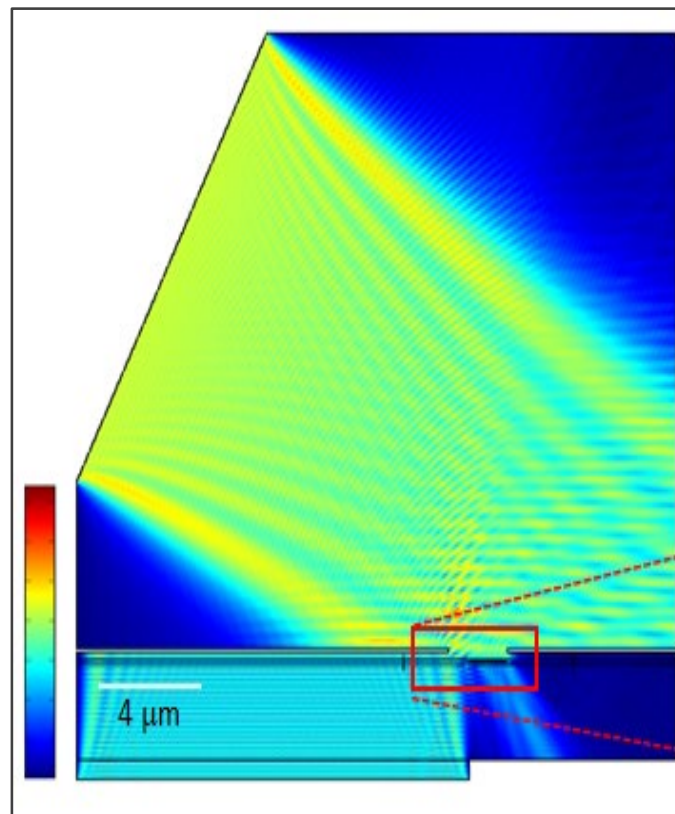

(a)

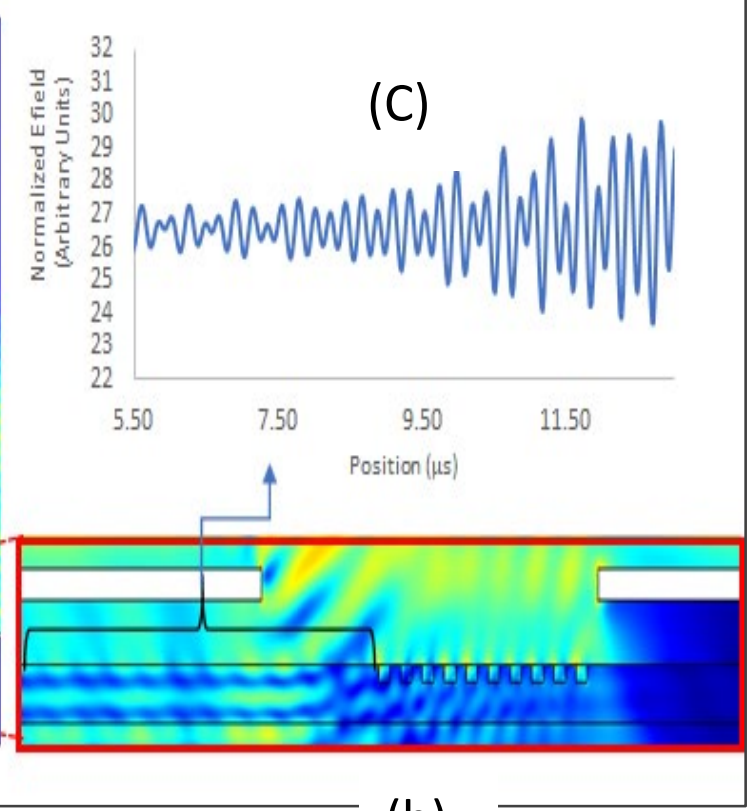

(b)

Figure 6. 2: (a) The oblique beam incident at $60^{\circ}$ angle on the grating etched on the ITO surface in the air medium that excites a single mode that interferes with the normal beam incident on the bottom surface of ITO in glass medium (b) Close up of the ITO layer showing interference pattern created by the guided mode and the normal beam on the left side of the grating. (C) Line graph of the electric field distribution of the interference pattern on the ITO surface extracted from the left side of the grating. 


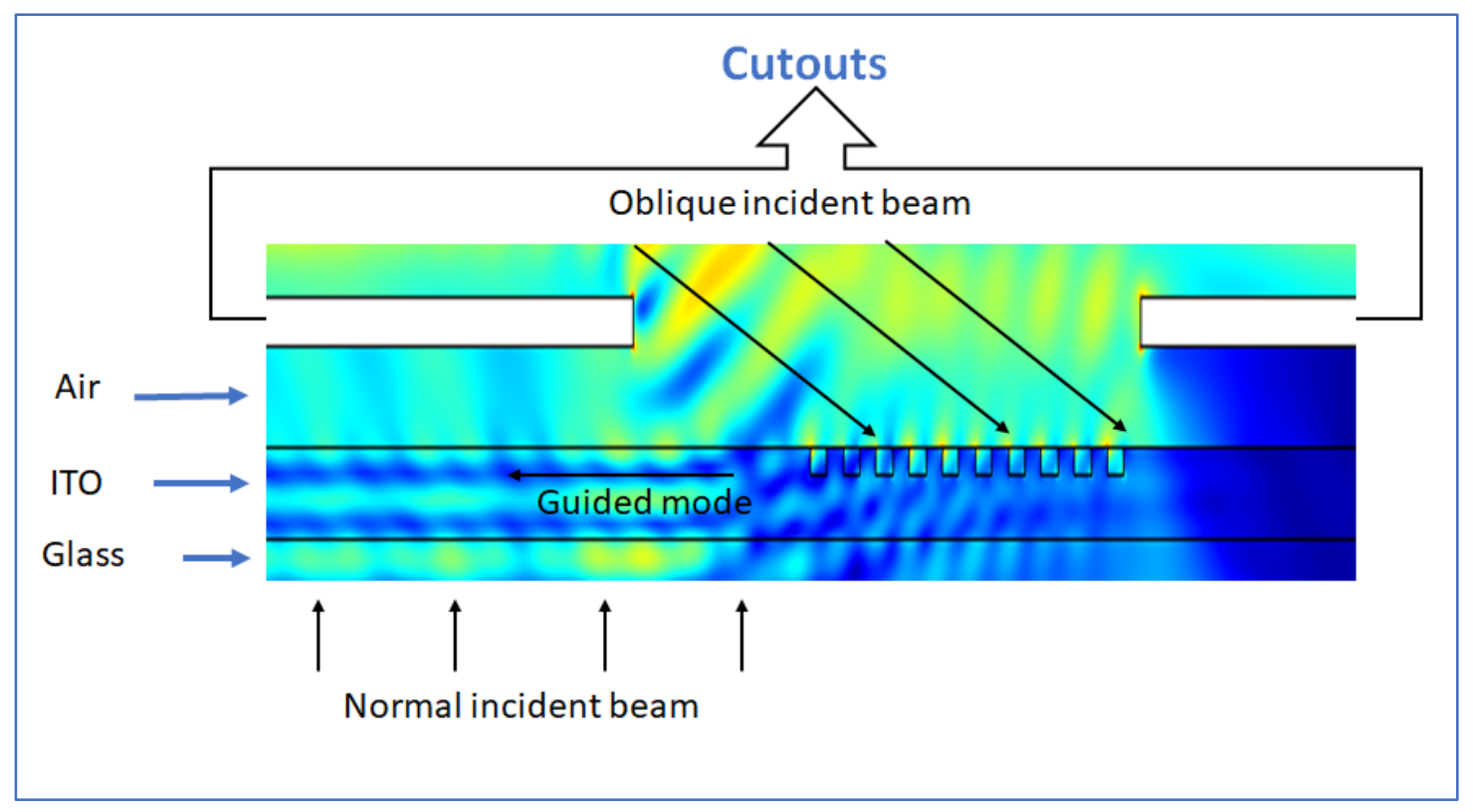

Figure 6.2 In the COMSOL model of the sample two rectangular shapes has been cut out (with no medium assigned to it) in the air medium to simulate obstacle preventing interference between the obliquely incident beam and the guided mode.

When the sample is illuminated with the oblique and normal beam, both of them will interfere with the excited guided mode. To analyse the interference of the mode with the normal beam only, a cut out is introduced above the ITO surface to prevent the interference between the ITO layer retaining the guided mode and the oblique beam as shown in figure 6.2. Experimentally this can be done by adjusting the laser spot position on the sample surface to allow the oblique beam to illuminate the grating only to excite the mode into ITO and prevent the oblique beam to illuminate the part of ITO where the guided mode propagates. This is done so that the interference movie created by the 
superposition between the mode and the normal beam does not get obscured by the interference pattern created by the superpositon between the mode and the oblique beam.

\subsection{Fourier Transform Verifying Mode Coupling}

The Fourier transform of the spatial electric field distribution shows peaks that correspond to the interference spacing created by the guided mode and an incident beam it interferes with. We analyze the Fourier transform of the normalized electric field distribution for the linear single photon photoemission process.

This spacing of the interference pattern at the surface of the ITO layer can be calculated for the linear case $(n=1)$ from the following equation showing relations between the wave vectors of the mode, incident beam and the interference pattern,

$$
\boldsymbol{k}_{\text {int }}=\mathbf{k}_{\mathrm{eff}}-\mathbf{k}_{\text {nor }}
$$

The wave vector of interference pattern, $\boldsymbol{k}_{\boldsymbol{i n t}}=\frac{2 \pi}{\mathrm{d}}$. The above equation can be used to determine the interference spacing of the superposition pattern yielded by the mode with wave vector $\mathbf{k}_{\text {eff }}$ and the normally incident pump beam with wave vector $\mathbf{k}_{\text {nor. }}^{\mid}$. The wavevector of the mode is related to its effective refractive index as,

$$
\mathrm{K}_{\mathrm{eff}}=\mathrm{kn} \mathrm{n}_{\mathrm{eff}}=\frac{2 \pi}{\lambda} \mathrm{n}_{\mathrm{eff}}
$$


Here, $\mathrm{k}$ is the wave number of incident beam in the air. The wave vector of the normally incident beam is $\mathrm{k}_{\mathrm{nor}}^{\mid} \mathrm{I}=\mathrm{k} \sin \theta$ which vanishes as for $\theta=0$. Hence, we have,

$$
\frac{2 \pi}{d}=\frac{2 \pi}{\lambda} n_{\text {eff }} \quad \text { or } d=\frac{\lambda}{n_{\text {eff }}}
$$

So, when the mode with effective index 1.885 interferes with the normally incident pump beam, their interference spacing will be,

$$
\mathrm{d}=\frac{410}{1.885} \mathrm{~nm}=217 \mathrm{~nm}=0.217 \mu \mathrm{m} .
$$

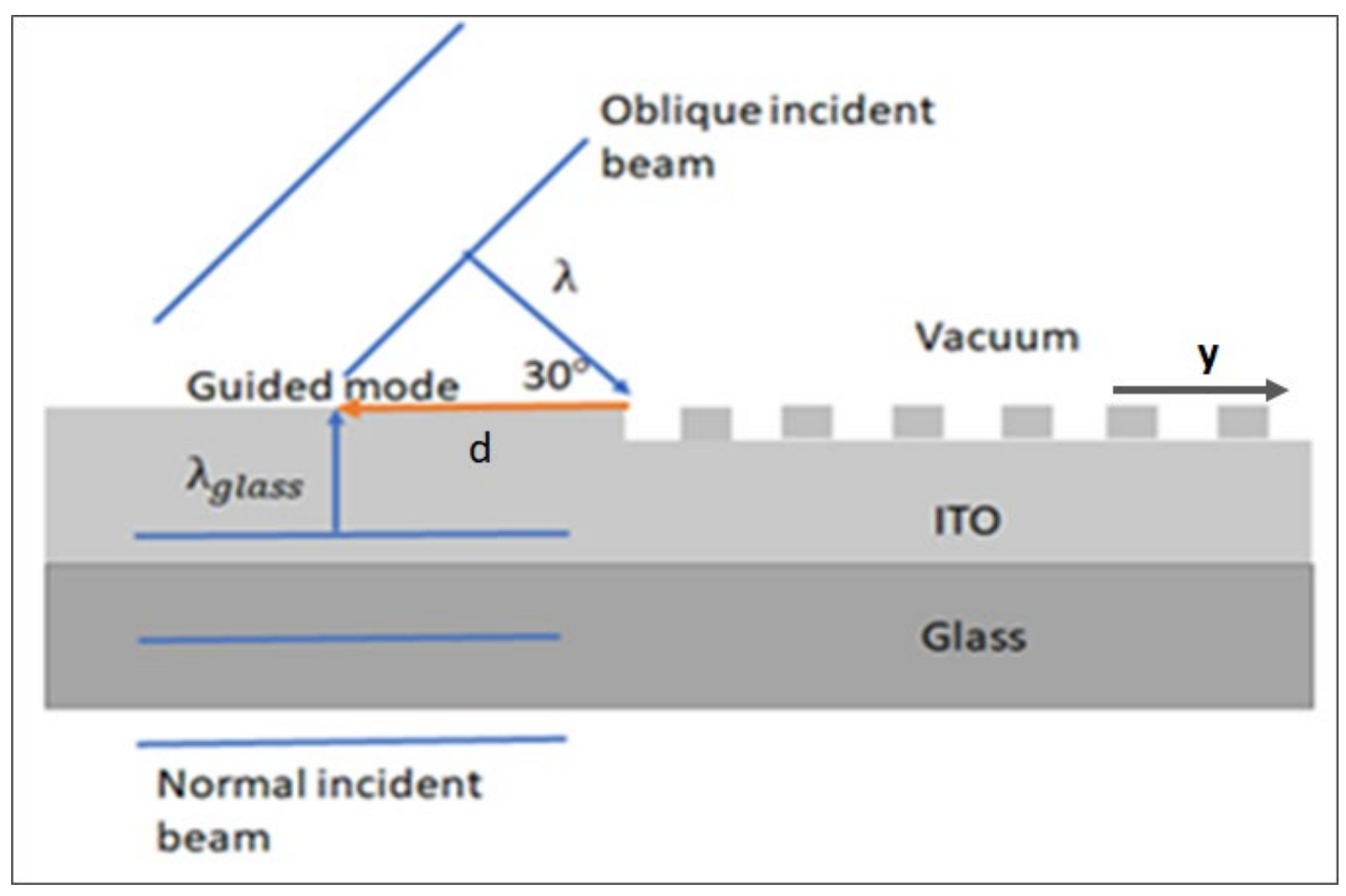

Figure 6.3: The wave front of the obliquely incident beam upon incident on the grating excites the guided mode that interferes with the wave front of the normally incident beam. 
The Fourier transform of the electric field distribution of the interference pattern across a line when the sample is illuminated with both the oblique and the normal beam shows a peak at the interference spacing $0.217 \mu \mathrm{m}$. The appearance of the peak at the interference spacing that matches the theoretically calculated spacing verifies the coupling of the guided mode of effective refractive index 1.885 into the waveguide. The Fourier transform of the line field distribution is also obtained for illumination of the sample with only the normal beam. This FFT periodogram also shows peak at the spacing $0.217 \mu \mathrm{m}$. Thus, the normal beam also excites the mode into the waveguide, but by comparing the Fourier peaks we can see this coupling is weaker in comparison to the mode excitation with the oblique beam.

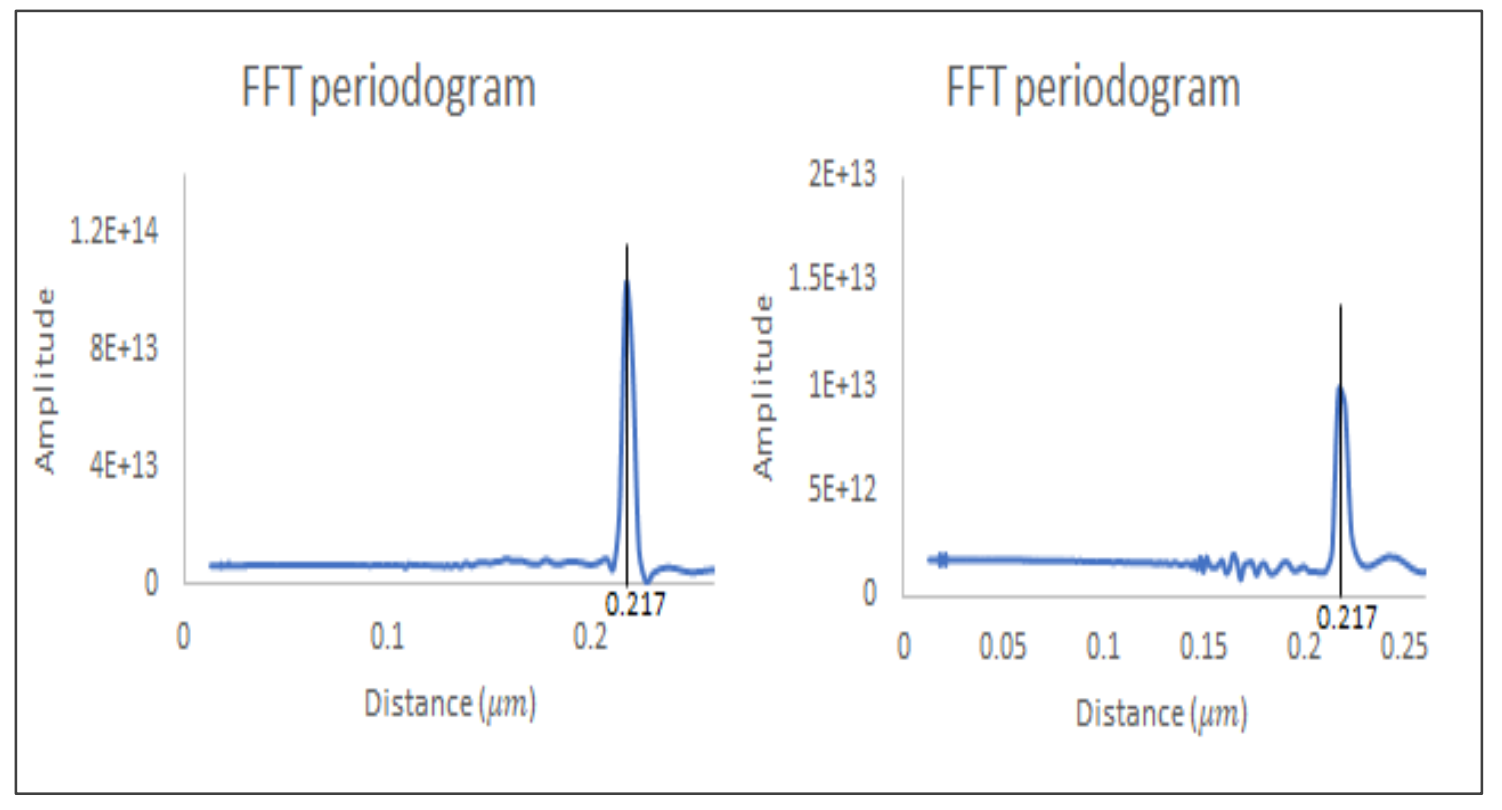

Figure 6.4: Fourier transform of normalized electric field distribution extracted for (a) both normal and oblique beam incident on the sample where the oblique beam only excites 
the guided mode and only the normal beam interfering with the mode and (b) only the normal beam incident on the sample exciting the mode as well as interfering with it.

\subsection{Grating length, depth and coupled power}

While the grating period is adjusted to couple a specific mode into the waveguide, the depth of the grating and the number of the groves are adjusted to vary the coupled power. The oblique beam is used to excite the mode and the grating parameters are determined to achieve maximum coupled power.

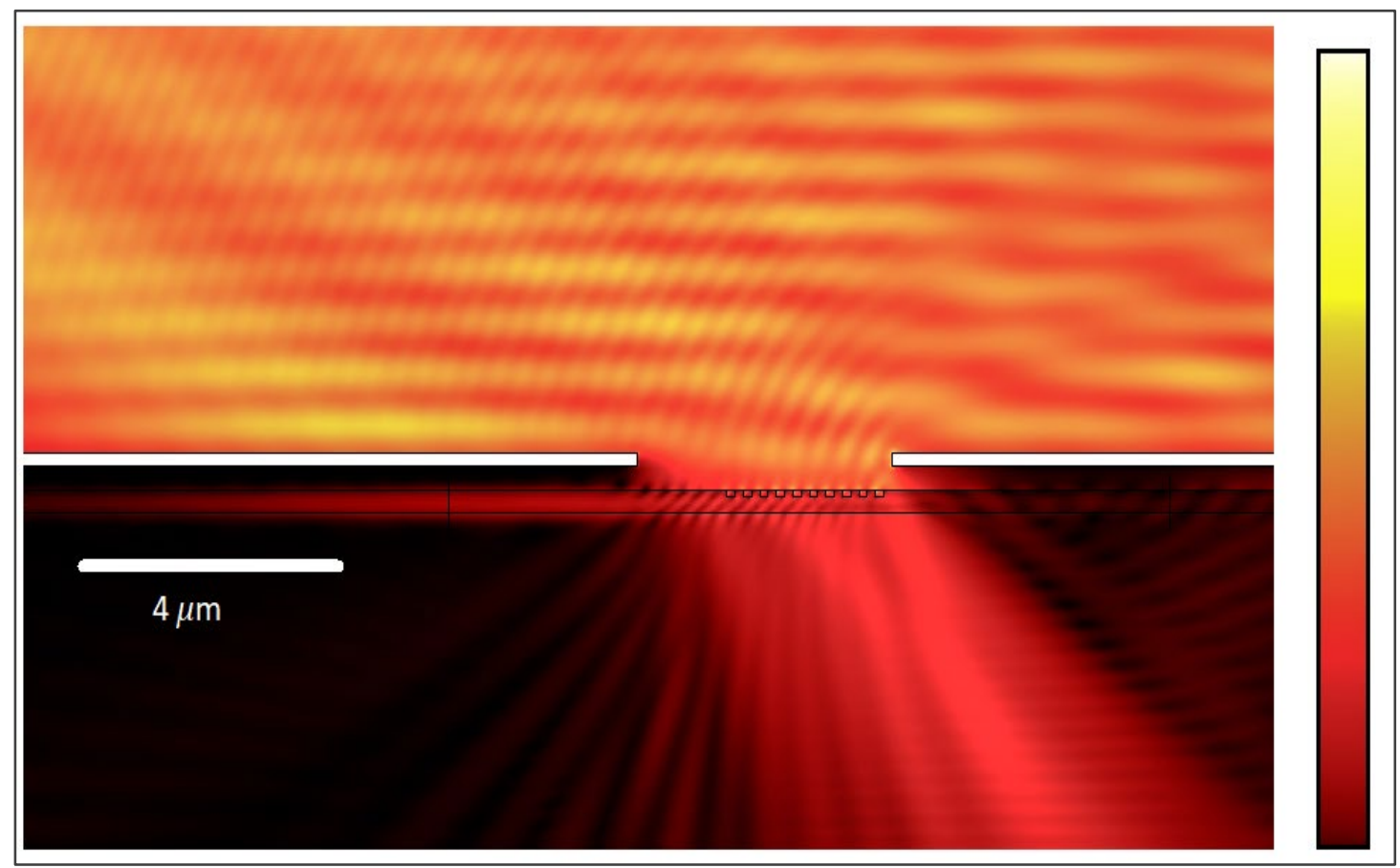

Figure 6. 5: The oblique beam incident on the optimized grating coupler excites a guided mode that propagates away from the grating within the waveguide.

The variation of coupled power with depth and size were numerically calculated using COMSOL. For different grating depths the grating size is varied from 1 (single 
trench) to 40 . For each depth value the coupled power increases with grating size to a maximum value and decreases with farther increase in size. It is found that for a binary grating $40 \mathrm{~nm}$ grating depth gives the maximum coupled power with grating size being 20.

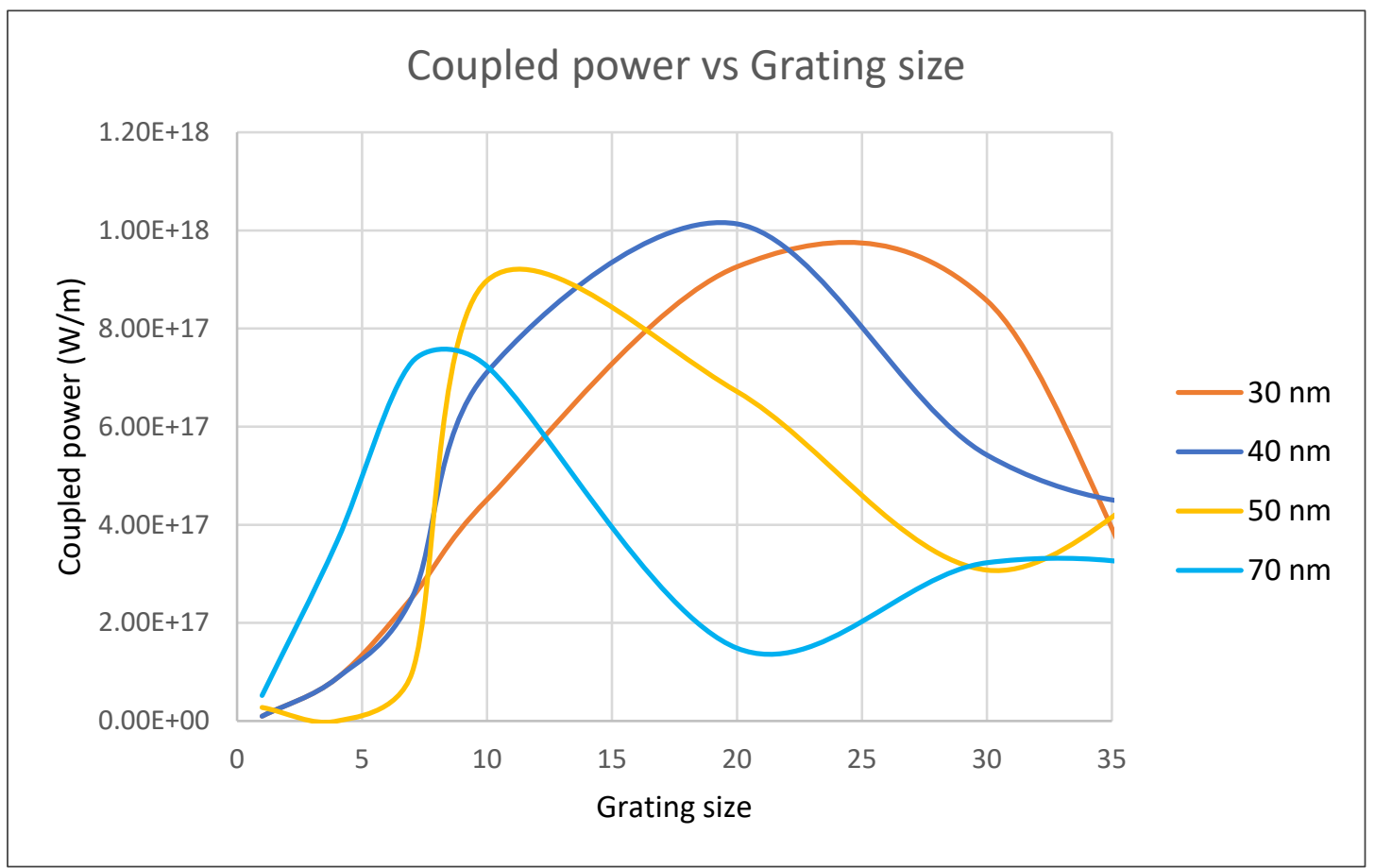

Figure 6. 6: graph showing the variation of coupled power with grating size at different grating depths.

\subsection{Grating Shape and Coupled Power}

To evaluate the dependence of coupled power on the grating shape, maximum coupled power for a binary grating and two types of blazed gratings are obtained. One of the two types of blaze grating evaluated has the slanted surface facing toward the oblique beam (left) and the other type with slanted surface facing away from it (right). For both type of blazed gratings, the maximum coupled power is observed with grating depth 70 
$\mathrm{nm}$ and size 10.

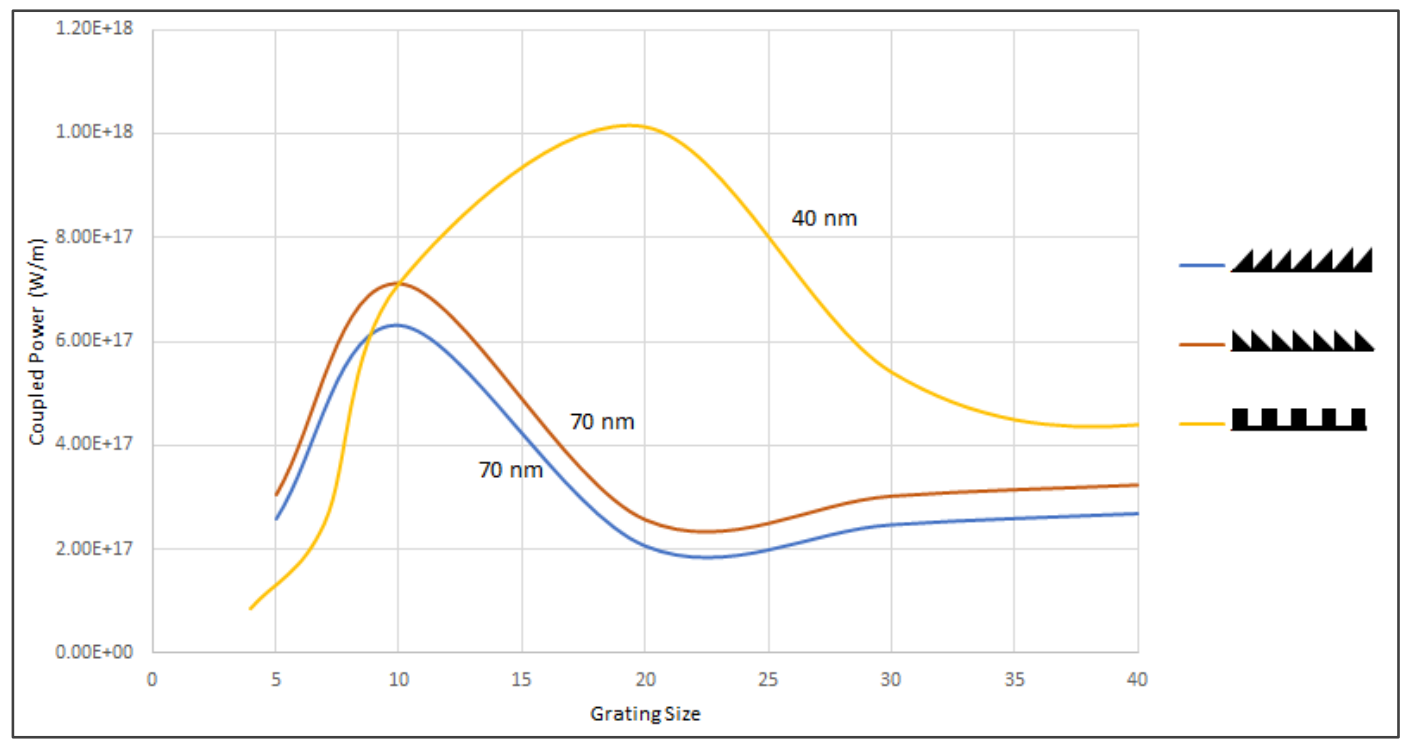

Figure 6. 7: graph showing comparison of coupled power for different grating shapes.

The depths are chosen for each shape to achieve maximum coupled power.

\subsection{Phase Delay and Interference Pattern Position}

As the delay between the pump and probe pulse are varied, the mode and the probe pulse meet farther and farther away from the grating edge causing the interference pattern to move away from that edge. This time evolution is recorded by varying the delay between the oblique and the normal beam in steps of $\frac{\pi}{2}$ for phase delay and $0.34 \mathrm{fs}$ in time delay for one optical cycle of the excitation laser. The resultant normalized electric field distribution on the surface and inside the ITO layer for each delay step is shown in figure 6. 


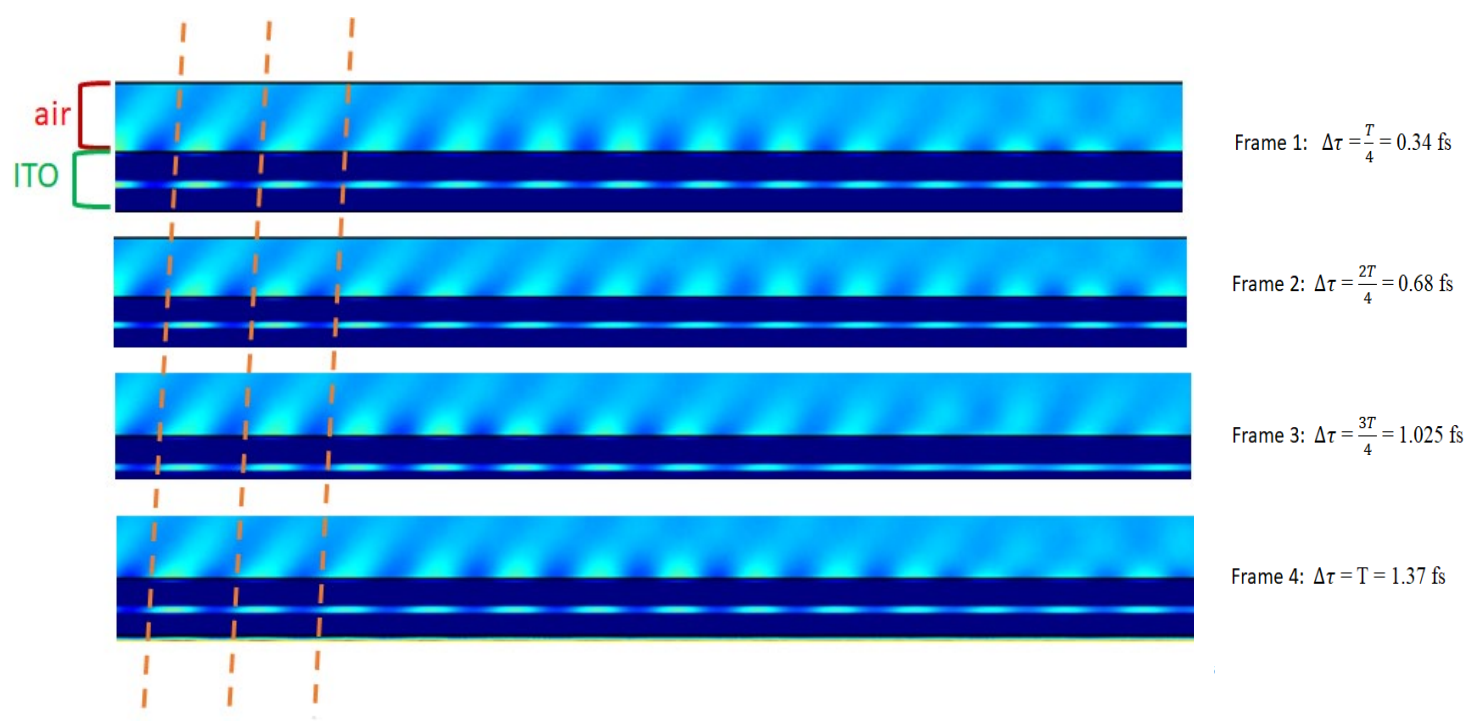

Figure 6.8: Simulated image of electric field distribution on the ITO surface as well as within the ITO layer. Each dashed line shows the edge of the same peak progressing with increased time delay.

The line electric field distribution across the ITO surface is extracted for each delay steps to calculation the velocity of the peaks. The time progression of each peak $\frac{\Delta x}{\Delta \tau}$ of the electric field distribution is related to light velocity in vacuum by the relation,

$$
\begin{gathered}
n_{\text {eff }}-\sin \theta=c \frac{\Delta x}{\Delta \tau} \\
\text { Or } \frac{\Delta x}{\Delta \tau}=\frac{\frac{c}{n_{e f f}}}{1-\frac{\sin \theta}{n_{e f f}}}=\frac{3 \times \frac{10^{8}}{1.885}}{1-\frac{\sin 0}{1.885}}=1.59 \times 10^{8} \frac{\mathrm{m}}{\mathrm{s}}
\end{gathered}
$$

The progression of any peak of the simulated electric field distribution with $\Delta \tau=0.34 \mathrm{fs}$ time delay increment between the pump and probe beam is found to be $\Delta x=0.054 \mu \mathrm{m}$ or 


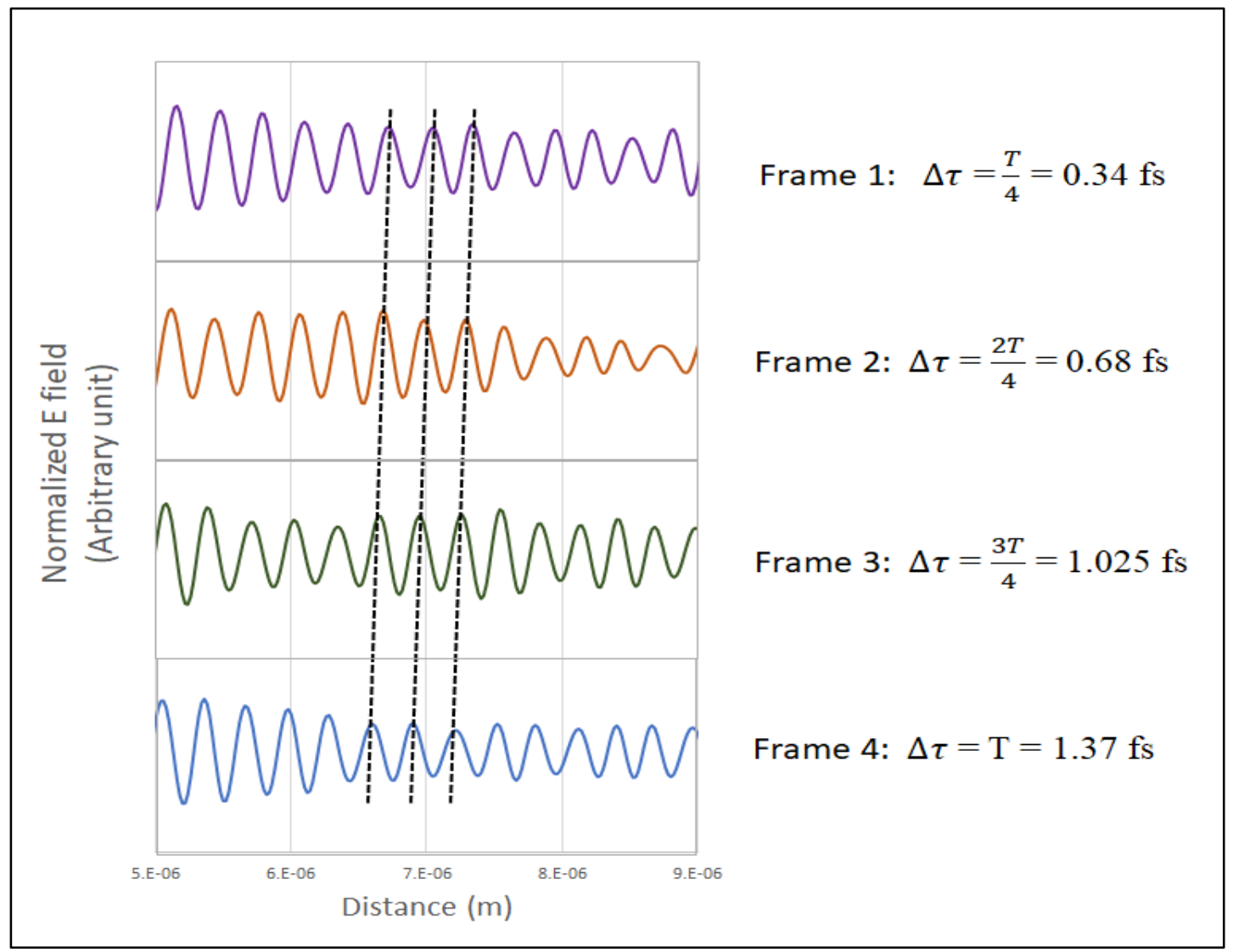

Figure 6. 9: Line electric field distribution for each delay steps across the ITO surface showing time progression of the electric field distribution peaks. Each dashed line indicates the same peak advancing in distance in each successive frame.

Hence the speed is,

which agrees with the theoretical value found in (6.13).

$$
\frac{\Delta x}{\Delta \tau}=\frac{0.054 \mu \mathrm{m}}{0.34 \mathrm{fs}}=\frac{0.054 \times 10^{-6}}{0.34 \times 10^{-15}} \mathrm{~m} / \mathrm{s}=1.588 \times 10^{8} \mathrm{~m} / \mathrm{s}
$$

When imaged by PEEM the intensity distribution on the ITO surface will be proportional to the fourth power of corresponding E field as discussed earlier of in this 
chapter. This intensity distribution will show the interference pattern yielded by the superposition of the guided mode and normally incident beam on the ITO surface for a specific delay between the obliquely (pump pulse) and normally (probe pulse) incident beam. This interference pattern will consist two different wave numbers and hence two different interference spacings. And as the delay between the pump and probe pulse is increased from $\mathrm{T} / 4$ to $\mathrm{T}$ the imaged interference peaks will move a distance at the speed of electromagnetic wave within ITO i.e. $1.59 \times 10^{8} \mathrm{~m} / \mathrm{s}$. 


\section{Conclusion}

In this study we have simulated the PEEM images of ITO surface which is simultaneously illuminated by a pump and probe beam. An efficient grating coupler is also designed through simulation to enable the obliquely incident pump beam to excite a guided mode into the waveguide. This guided mode is probed by a normally incident beam that interferes with the guided mode and creates a pattern of varying intensity on the waveguide surface. This interference pattern can be studied to characterize the mode propagations as well as the waveguide. Such pump and probe method is similar to Mach Zehnder interferometry except using Mach Zehnder interferometer we send a light wave through the sample and evaluate it after it comes out of the other side of the sample while using PEEM we can evaluate the light wave while it is inside the sample.

By changing the delay between the pump and the probe beam the observed interference pattern on the ITO surface changes its position. By gradually increase the time delay a movie-like documentation of the light propagation in the waveguide is obtained. The simulations show the interference maxima peaks to move away from the coupler with the speed of light for an ITO waveguide. This speed is $\mathrm{c} / \mathrm{n}_{\text {eff. }}$ In experimental PEEM many experimental effects could be explored, such as localized scattering, boundary effects, secondary excitations within or outside the waveguide, coupling to molecules, surface plasmons, dephasing and energy dispersions etc. Analyzing the actual light propagation on a nanometer scale is of interest for many applications in the photonics field. 
Beyond these practical aspects we would like to point to the intricate interpretation of these experiments. The photoelectrons ejecting from the sample surface carry information about its interaction with one or more incident photons. This information includes how much energy it has acquired from the interaction with the light quanta. PEEM image of this energy distribution of the photoelectrons ejected from different parts of the sample surface shows periodic intensity variation on the surface due to the interference between the incident light and the guided mode. Thus, photoelectric effect- a purely quantum phenomena allows PEEM to image the interference pattern caused by the classical wave nature of light. Such simultaneous observation of classical and quantum phenomenon enables PEEM in a unique way to study the properties of the confined light as well as the confining materials. 


\section{References}

[1] Ostrowsky, D. B., Allibart, O., Baldi, P. A., \& Tanzilli, S. (2004). Photonic integrated circuits for quantum communication and computation. Integrated Optics and Photonic Integrated Circuits. doi: 10.1117/12.549918

[2] Chandrasekar, R., Lapin, Z. J., Nichols, A. S., Braun, R. M., \& Fountain, A. W. (2019). Photonic integrated circuits for Department of Defense-relevant chemical and biological sensing applications: state-of-the-art and future outlooks. Optical Engineering, 58(02), 1. doi: 10.1117/1.oe.58.2.020901

[3] Chovan, J., \& Uherek, F. (2018). Photonic Integrated Circuits for Communication Systems. Radioengineering, 27(2), 357-363. doi: 10.13164/re.2018.0357

[4] S. Meister et.al (2012). "Photonic Integrated Circuits for Optical Communications." Optoelectronics, 7 (2).

[5] Passaro et al. (2012). "Recent Advances in Integrated Photonics Sensors." Sensors $12 \mathrm{~m} \mathrm{15558-15598}$

[6] Chen, C., \& Wang, J. (2020). Optical biosensors: an exhaustive and comprehensive review. The Analyst, 145(5), 1605-1628. doi: 10.1039/c9an01998g [7] $5.4 \mathrm{~nm}$ spatial resolution in biological photoemission electron microscopy

[8] Schmidt, T., Sala, A., Marchetto, H., Umbach, E., \& Freund, H.-J. (2013). First experimental proof for aberration correction in XPEEM: Resolution, transmission enhancement, and limitation by space charge effects. Ultramicroscopy, 126, 23-32. doi: 10.1016/j.ultramic.2012.11.004

[9] Lemke, Christoph \& Leißner, Till \& Jauernik, Stephan \& Klick, Alwin \& Fiutowski, Jacek \& Kjelstrup-Hansen, Jakob \& Rubahn, Horst-Günter \& Bauer, M. (2012). Mapping surface plasmon polariton propagation via counter-propagating light pulses. Optics express. 20. 12877-84. 10.1364/OE.20.012877.

[10] J. P. S. Fitzgerald, R. C. Word, and R. Könenkamp, "Subwavelength visualization

of light in thin film waveguides with photoelectrons," Phys. Rev. B - Condens. Matter Mater. Phys., vol. 89, no. 19, 2014.

[11] Fitzgerald, J. P. S., et al. "Subwavelength Visualization of Light in Thin Film Waveguides with Photoelectrons." Physical Review B, vol. 89, no. 19, 2014, doi:10.1103/physrevb.89.195129.

[12] Fitzgerald, J. P. S., et al. "Photonic Near-Field Imaging in Multiphoton Photoemission Electron Microscopy.” Physical Review B, vol. 87, no. 20, 2013, doi:10.1103/physrevb.87.205419. 
[13] König, Tobias A. F., et al. "Electrically Tunable Plasmonic Behavior of Nanocube-Polymer Nanomaterials Induced by a Redox-Active Electrochromic Polymer." ACS Nano, vol. 8, no. 6, 2014, pp. 6182-6192., doi:10.1021/nn501601e.

[14] R. Könenkamp, R. C. Word, G. F. Rempfer, T. Dixon, L. Almaraz, and T. Jones,

"5.4Nm Spatial Resolution in Biological Photoemission Electron Microscopy," Ultramicroscopy, vol. 110, no. 7, pp. 899-902, 2010.

[15] Gierster, L., et al. "A Sample Holder with Integrated Laser Optics for an ELMITEC

Photoemission Electron Microscope.” Review of Scientific Instruments, vol. 86, no. 2, 2015, p.

023702., doi:10.1063/1.4907402.

Dielectric

[16] T. A. Stenmark, "Photoemission Electron Microscopy for Analysis of

Structures and the Goos-Hänchen Shift,” Portland State University, 2016.

[17] Word, Robert C., and Rolf Könenkamp. "Photonic and Plasmonic Surface Field Distributions Characterized with Normal- and Oblique-Incidence Multi-Photon PEEM." Ultramicroscopy, vol. 183, 2017, pp. 43-48., doi:10.1016/j.ultramic.2017.05.012

[18] Boudrioua, A. (2009). Photonic waveguides: Theory and applications. London, UK:

ISTE.

[19] Harvey, J. E., \& Pfisterer, R. N. (2019). Understanding diffraction grating behavior: Including conical diffraction and Rayleigh anomalies from transmission gratings. Optical Engineering, 58(08), 1. doi:10.1117/1.oe.58.8.087105

[20] Herzig, H. P. (1997). Micro-optics: Elements, systems and applications. London: Taylor \& Francis. 2010

[21] Jin, Jian- Ming. Theory and Computation of Electromagnetic Fields. Oct.

[22] H. Whitney, Geometric integration theory, Princeton UP, Princeton, 1957.

[23] A. Nentchev, Numerical analysis and simulation in microelectronics by vector finite elements, 2008.

[24] Jin, Jian-Ming. The Finite Element Method in Electromagnetics. John Wiley \& Sons Inc., 2014. 
[25] Comsol AB. Comsol Multiphysics, RF Module User's Guide, version 4.3. 2012. 1998 ,

[26] Oven, S. A Survey of Unstructured Mesh Generation Technology.IMR,

[27] George, P., Frey, S. Mesh Generation. 2nd ed. Hoboken, Wiley, 2008.

[28] Zhu, Y., Cangellaris, A. Multigrid Finite Element Methods for Electromagnetic Field Modeling. Hoboken, New Jersey, US, John Wiley \& Sons, 2006.

[29] M. L. Barton and Z. J. Cendes, New vector finite elements for threedimensional magnetic field computation, J. Appl. Phys., vol. 61, pp. 3919-3921, 1987. IEEE [30] Z. J. Cendes, Vector finite elements for electromagnetic field computations,

Trans. Magn., vol. MAG-27, no. 5, pp. 3958-3966, Sept. 1991. Magn.,

[31] J. P. Webb, Edge elements and what they can do for you, IEEE Trans. vol. MAG-29, pp. 1460-1465, Mar. 1993.

[32] G. Mur, The finite-element modeling of three-dimensional electromagnetic fields using edge and nodal elements, IEEE Trans. Antennas Propag., vol. 41, no. 7, pp. 948-953

[33] Feng, J., \& Zhou, Z. (2006). High efficiency compact grating coupler for integrated optical circuits. Proceedings of SPIE. doi:10.1117/12.688732

[34] Dakss, M., Kuhn, L., Heidrich, P., \& Scott, B. (1970, June 15). Grating coupler for efficient excitation of optical guided waves in thin films. https://aip.scitation.org/doi/10.1063/1.1653091

[35] Könenkamp, R., Word, R. C., Rempfer, G., Dixon, T., Almaraz, L., \& Jones, T. (2010). $5.4 \mathrm{~nm}$ spatial resolution in biological photoemission electron microscopy. Ultramicroscopy, 110(7), 899-902. doi: 10.1016/j.ultramic.2010.04.005 